ESAIM: COCV 22 (2016) 519-538

DOI: $10.1051 / \mathrm{cocv} / 2015016$
ESAIM: Control, Optimisation and Calculus of Variations

www.esaim-cocv.org

\title{
FULLY COUPLED FORWARD-BACKWARD SDES INVOLVING THE VALUE FUNCTION AND ASSOCIATED NONLOCAL HAMILTON-JACOBI-BELLMAN EQUATIONS *
}

\author{
TAO HAO ${ }^{1,2}$ AND JUAN Li ${ }^{1}$
}

\begin{abstract}
A new type of controlled fully coupled forward-backward stochastic differential equations is discussed, namely those involving the value function. With a new iteration method, we prove an existence and uniqueness theorem of a solution for this type of equations. Using the notion of extended "backward semigroup", we prove that the value function satisfies the dynamic programming principle and is a viscosity solution of the associated nonlocal Hamilton-Jacobi-Bellman equation.
\end{abstract}

Mathematics Subject Classification. 60H10,60H30, 35K65.

Received February 24, 2014. Revised November 24, 2014

Published online April 6, 2016.

\section{INTRODUCTION}

The main purpose of this work is to establish a new type of controlled fully coupled forward-backward stochastic differential equations (FBSDEs), namely those involving the value function. We prove the existence and uniqueness of a solution for this new type of equations and we study the related nonlocal second-order quasi-linear Hamilton-Jacobi-Bellman (HJB) equations. Our work is inspired by the recent developments in mean-field stochastic differential equations (SDEs), mean-field backward SDEs (BSDEs) and fully coupled mean-field FBSDEs (see $[5,6,12]$, etc.), and Hao and Li ([9]). Another motivation comes from the study of control problems in the mean-field framework and the investigation of the associated nonlocal partial differential equations of HJB type.

Models of large stochastic particle systems with mean-field interaction can be met in various fields such as statistical mechanics and physics, quantum mechanics and quantum chemistry. Mean-field problems have been studied by many authors (see, e.g., $[2,3,7,11,18,19]$ ). Due to these works we know that linear McKean-Vlasov PDEs can be interpreted stochastically. In 2009, Buckdahn et al. [6] investigated in a purely stochastic approach

\footnotetext{
Keywords and phrases. Fully coupled FBSDE involving value function, dynamic programming principle, fully coupled mean-field FBSDE, viscosity solution, nonlocal Hamilton-Jacobi-Bellman equation.

* Juan Li is the corresponding author. The work has been supported by the NSF of P.R.China (Nos. 11071144, 11171187, 11222110), Shandong Province (Nos. BS2011SF010, JQ201202), SRF for ROCS (SEM), Program for New Century Excellent Talents in University (No. NCET-12-0331), 111 Project (No. B12023).

1 School of Mathematics and Statistics, Shandong University, Weihai, Weihai 264209, P.R. China.

2 School of Science and Technology, Shandong University of Traditional Chinese Medicine, Jinan 250355, P.R. China.

juanli@sdu.edu.cn; haotao2012@hotmail.com
} 
fully nonlinear BSDEs of mean-field type, called mean-field BSDEs. In [5], Buckdahn et al. deepened the study of mean-field BSDEs and gave a probability interpretation for the associated second-order quasi-linear nonlocal partial differential equations (PDEs).

On the other hand, Antonelli [1] was the first to study the existence and the uniqueness for fully coupled FBSDEs on a small time interval; he pointed out that the Lipschitz assumption is not sufficient for the existence of the solution of fully coupled FBSDEs over an arbitrarily large time interval. Since then, the theory of fully coupled FBSDEs has been developed very quickly. For instance, with PDE approaches combined with stochastic methods, Ma et al. [15] showed the existence and the uniqueness for a type of fully coupled FBSDEs which forward SDEs are non-degenerate. Another direction of generalization was considered by $\mathrm{Hu}$ and Peng [10]: Under suitable monotonicity conditions they obtained the existence and the uniqueness for fully coupled FBSDEs on an arbitrarily large time interval, but with forward and backward equation being of the same dimension. In 1999, Peng and $\mathrm{Wu}$ [17] overcame this strict restriction by considering a multiplicative full-rank matrix $G$ with which one solution of the forward equation is multiplied, and they proved the existence and the uniqueness for general fully coupled FBSDEs under weaker monotonicity assumptions and discussed applications in optimal control. Li and Wei [13] considered optimal control problems for fully coupled FBSDEs and the associated second-order quasi-linear HJB equations. Unlike [13] we study the optimal control problem of fully coupled mean-field FBSDEs, and the associated non-local HJB equation is mean-field type. Recently, fully coupled mean-field FBSDEs were discussed by Min et al. [12]. The authors gave an existence and uniqueness theorem of a solution for this type of equations by using a continuation method.

In [5] their original motivation is to give the stochastic interpretation of nonlocal PDE of mean-field type. However, the formally associated SDE does not generate a flow. To overcome this difficulty in [5] the initial condition was partially frozen, which leads to a system composed of decoupled mean-field FBSDE and a classical decoupled FBSDE depending on the solution of the mean-field SDE. In this paper we adopt and extend this approach to control problems, in order to give stochastic interpretation to nonlocal Hamilton-Jacobi-Bellman equations of mean-field type. More precisely, let $x_{0} \in \mathbb{R}^{n}$ and $\bar{v} \in \mathcal{V}_{0, T}$ be arbitrary but fixed, and let $x \in \mathbb{R}^{n}$, $t \in[0, T], v \in \mathcal{V}_{t, T}$, we consider the following fully coupled FBSDE:

$$
\left\{\begin{aligned}
\mathrm{d} X_{s}^{t, x ; v}= & E^{\prime}\left[b\left(s,\left(X_{s}^{0, x_{0} ; \bar{v}}\right)^{\prime}, W\left(s,\left(X_{s}^{0, x_{0} ; \bar{v}}\right)^{\prime}\right), X_{s}^{t, x ; v}, Y_{s}^{t, x ; v}, Z_{s}^{t, x ; v}, v_{s}\right)\right] \mathrm{d} s \\
& +E^{\prime}\left[\sigma\left(s,\left(X_{s}^{0, x_{0} ; \bar{v}}\right)^{\prime}, W\left(s,\left(X_{s}^{0, x_{0} ; \bar{v}}\right)^{\prime}\right), X_{s}^{t, x ; v}, Y_{s}^{t, x ; v}, v_{s}\right)\right] \mathrm{d} B_{s}, \\
\mathrm{~d} Y_{s}^{t, x ; v}= & -E^{\prime}\left[f\left(s,\left(X_{s}^{0, x_{0} ; \bar{v}}\right)^{\prime}, W\left(s,\left(X_{s}^{0, x_{0} ; \bar{v}}\right)^{\prime}\right), X_{s}^{t, x ; v}, Y_{s}^{t, x ; v}, Z_{s}^{t, x ; v}, v_{s}\right)\right] \mathrm{d} s \\
& +Z_{s}^{t, x ; v} \mathrm{~d} B_{s}, \\
X_{t}^{t, x ; v}= & x, \quad Y_{T}^{t, x ; v}=E^{\prime}\left[\Phi\left(\left(X_{T}^{0, x_{0} ; \bar{v}}\right)^{\prime}, X_{T}^{t, x ; v}\right)\right], \\
W(t, x)= & \underset{v \in \mathcal{V}_{t, T}}{\operatorname{essinf}} Y_{t}^{t, x ; v},(t, x) \in[0, T] \times \mathbb{R}^{n},
\end{aligned}\right.
$$

where the essential infimum is taken over all admissible stochastic control processes $v$ over the time interval $[t, T]$. By $\left(X^{0, x_{0} ; \bar{v}}\right)^{\prime}=\left(X_{s}^{0, x_{0} ; \bar{v}}\right)_{s \in[t, T]}^{\prime}$ we denote an independent and identically distributed copy of the process $X^{0, x_{0} ; \bar{v}}=\left(X_{s}^{0, x_{0} ; \bar{v}}\right)_{s \in[t, T]}$ which is supposed to be independent of the driving Brownian motion $B$ and, thus also of all processes $X^{0, x_{0} ; \bar{v}}, X^{t, x ; v}, Y^{t, x ; v}, Z^{t, x ; v}$, and $\bar{v}, v$ adapted with respect to the filtration generated by $B$. By $E^{\prime}$ [] we denote an expectation concerning only $\left(X^{0, x_{0} ; \bar{v}}\right)^{\prime}$ but not the other processes (for more details the reader is referred to [5]). In order to solve (1.1) involving the value function of the control problem itself, we replace first $W$ by any function $\tilde{W}(s, x)$, which is Lipschitz and of linear growth in $x$, and under suitable monotonicity conditions we show the existence and the uniqueness of a solution $\left(X^{t, x ; v}, Y^{t, x ; v}, Z^{t, x ; v}\right)$ of $(1.1)$, for all $(t, x, v)$ (and so, in particular, for $(t, x, v)=\left(0, x_{0}, \bar{v}\right)$. In fact, beginning with choosing $(t, x, v)=\left(0, x_{0}, \bar{v}\right)$ we find $\left(X^{0, x_{0} ; \bar{v}}, Y^{0, x_{0} ; \bar{v}}, Z^{0, x_{0} ; \bar{v}}\right)$ which allows then to consider arbitrary $(t, x, v)$ in (1.1)). Moreover, we will show that there exists a constant $L_{0}>0$ depending only on $L_{b}$ in (H3.3) and the Lipschitz constants of $b, \sigma, f$ and $\Phi$ in $(x, y, z)$ and $x$, such that the value function $W(t, x) \triangleq \underset{v \in \mathcal{V}_{t, T}}{\operatorname{essinf}} Y_{t}^{t, x ; v}$ is Lipschitz and of linear growth in $x$, both with a constant $L_{0}$ which is independent of $\tilde{W}$. This means that, no matter what the Lipschitz and the linear 
growth constants of $\tilde{W}$ are, there is a common constant $L_{0}>0$ such that $W(t, x)$ is Lipschitz and of linear growth in $x$ with a Lipschitz and growth constant $L_{0}$. With a new iteration method which is different from [9], beginning with $\tilde{W}=W^{0}=0$, we get four convergent sequences $\left\{X^{0, x_{0} ; \bar{v}, i}\right\},\left\{X^{t, x ; v, i}\right\},\left\{Y^{t, x ; v, i}\right\},\left\{Z^{t, x ; v, i}\right\}$ and the associated $W^{i+1}(t, x)=\underset{v \in \mathcal{V}_{t, T}}{\operatorname{essinf}} Y_{t}^{t, x ; v, i}$. We prove that all these sequences converge to the solution processes (Thm. 3.7). Once having the existence and the uniqueness of a solution for (1.1) we prove the DPP for the value function $W$. For this we use an extended "backward semigroup" (Thm. 4.2) generalizing Peng's original one [16]. Using this DPP, it becomes easy to check that $W(t, x)$ is Hölder continuous in $t$ (Prop. 4.4). Furthermore, we study the related nonlocal HJB equations associated with (1.1). Using a short and direct probabilistic argument based on results by $\mathrm{Li}$ and Wei [13], we prove that the value function $W$ is a viscosity solution of our nonlocal HJB equation (5.1) (Thm. 5.2). In Theorem 5.3 we show the uniqueness of viscosity solution of our nonlocal HJB equation (5.1) when $\sigma$ doesn't depend on $y$ with the help of the uniqueness of the solution of fully coupled FBSDE (1.1).

In comparison with [9] a main difficulty consists in the fact that we have to deal here with a fully coupled FBSDE: Any iteration procedure also involves the solution of the forward equation $X^{t, x ; v, i}$, and thus also $\left(X^{0, x_{0} ; \bar{v}, i}\right)^{\prime}$-the variable of $W^{i-1}$. In order to get the convergence of the iteration sequence we have to suppose that the coefficients $b\left(s, x^{\prime}, y^{\prime}, x, y, z, v\right), \sigma\left(s, x^{\prime}, y^{\prime}, x, y, v\right)$ and $f\left(s, x^{\prime}, y^{\prime}, x, y, z, v\right)$ are Lipschitz in $y^{\prime}$ with a sufficiently small Lipschitz constant.

Our paper is organized as follows. In Section 2, we recall some results on fully coupled mean-field FBSDEs, which are used frequently in what follows. Section 3 introduces the fully coupled FBSDEs involving value function and gives the proof of the existence and the uniqueness of solutions of equations. The DPP and the regularity of the value function are shown in Section 4. In Section 5, for this new type of equation we show that the value function is a unique viscosity solution of the associated nonlocal HJB equation. Some auxiliary lemmas are proved in Section 6.

\section{Fully COUPLED MEAN-FIELD FBSDES}

Our setting is that of the classical Wiener space: Let $T>0$ and $\Omega=C_{0}\left([0, T] ; \mathbb{R}^{d}\right)$ be the set of all continuous functions from $[0, T]$ to $\mathbb{R}^{d}$, beginning from $0 ; P$ is the Wiener measure under which the coordinate process $B$ : $B_{s}(\omega)=\omega_{s}, s \in[0, T], \omega \in \Omega$, is a $d$-dimensional Brownian motion. By $\mathcal{F}$ we denote the Borel $\sigma$-field over $\Omega$, completed by the set $\mathcal{N}$ of all $P$-null sets. We denote by $\mathbb{F}=\left\{\mathcal{F}_{s}, 0 \leq s \leq T\right\}$ the natural filtration generated by $\left\{B_{s}\right\}_{0 \leq s \leq T}$ and completed by $\mathcal{N}$.

We introduce the following spaces which will be often used:

- $\mathcal{S}_{\mathbb{F}}^{2}\left(0, T ; \mathbb{R}^{n}\right) \triangleq\left\{\left(\phi_{t}\right)_{0 \leq t \leq T} \mathbb{R}^{n}\right.$-valued $\mathbb{F}$-adapted càdlàg process : $\left.E\left[\sup _{0 \leq t \leq T}\left|\phi_{t}\right|^{2}\right]<+\infty\right\}$;

- $\mathcal{H}_{\mathbb{F}}^{2}\left(0, T ; \mathbb{R}^{n \times d}\right) \triangleq\left\{\left(l_{t}\right)_{t \in[0, T]} \mathbb{R}^{n \times d}\right.$-valued $\mathbb{F}$-progressively measurable process: $\left.\|l\|^{2}=E\left[\int_{0}^{T}\left|l_{t}\right|^{2} d t\right]<+\infty\right\}$.

Let us first recall some notations used for the mean-field framework and for the fully coupled mean-field FBSDEs in [12]; see also $[5,6]$.

By $(\bar{\Omega}, \overline{\mathcal{F}}, \bar{P})=(\Omega \times \Omega, \mathcal{F} \otimes \mathcal{F}, P \otimes P)$ we denote the product of $(\Omega, \mathcal{F}, P)$ with itself. The filtration in this space we consider is $\overline{\mathbb{F}}=\left\{\overline{\mathcal{F}}_{t}=\mathcal{F} \otimes \mathcal{F}_{t}, 0 \leq t \leq T\right\}$. By setting $\xi^{\prime}\left(\omega^{\prime}, \omega\right)=\xi\left(\omega^{\prime}\right),\left(\omega^{\prime}, \omega\right) \in \bar{\Omega}$, we can extend the real-valued random variables $\xi$ from $\Omega$ to $\bar{\Omega}$. For any integrable random variable $\theta \in L^{1}(\bar{\Omega}, \overline{\mathcal{F}}, \bar{P})$, $\theta(\cdot, \omega): \Omega \rightarrow \mathbb{R}$ is in $L^{1}(\Omega, \mathcal{F}, P), \mathrm{P}(\mathrm{d} \omega)$-a.s. Hence, we can define $E^{\prime}[\theta(\cdot, \omega)]=\int_{\Omega} \theta\left(\omega^{\prime}, \omega\right) P\left(\mathrm{~d} \omega^{\prime}\right)$. Using Fubini's Theorem, the expectation of $\theta$ can be computed as follows:

$$
\bar{E}[\theta]=\int_{\bar{\Omega}} \theta \mathrm{d} \bar{P}=\int_{\Omega} E^{\prime}[\theta(\cdot, \omega)] P(\mathrm{~d} \omega)=E\left[E^{\prime}[\theta]\right]
$$

Let $b\left(\bar{\omega}, t, x^{\prime}, y^{\prime}, z^{\prime}, x, y, z\right): \bar{\Omega} \times[0, T] \times \mathbb{R}^{n} \times \mathbb{R}^{m} \times \mathbb{R}^{m \times d} \times \mathbb{R}^{n} \times \mathbb{R}^{m} \times \mathbb{R}^{m \times d} \rightarrow \mathbb{R}^{n}$, $\sigma\left(\bar{\omega}, t, x^{\prime}, y^{\prime}, x, y\right): \bar{\Omega} \times[0, T] \times \mathbb{R}^{n} \times \mathbb{R}^{m} \times \mathbb{R}^{n} \times \mathbb{R}^{m} \rightarrow \mathbb{R}^{n \times d}$,

$f\left(\bar{\omega}, t, x^{\prime}, y^{\prime}, z^{\prime}, x, y, z\right): \bar{\Omega} \times[0, T] \times \mathbb{R}^{n} \times \mathbb{R}^{m} \times \mathbb{R}^{m \times d} \times \mathbb{R}^{n} \times \mathbb{R}^{m} \times \mathbb{R}^{m \times d} \rightarrow \mathbb{R}^{m}$, $\Phi\left(\bar{\omega}, x^{\prime}, x\right): \bar{\Omega} \times \mathbb{R}^{n} \times \mathbb{R}^{n} \rightarrow \mathbb{R}^{m}$ 
satisfy the following assumptions:

(H2.1) (i) There exist $L, \tilde{L}_{1}, \tilde{L}_{2}, \tilde{L}_{3}>0$ such that, for all $t \in[0, T], x_{1}, x_{1}^{\prime}, x_{2}, x_{2}^{\prime} \in \mathbb{R}^{n}, y_{1}, y_{1}^{\prime}, y_{2}, y_{2}^{\prime} \in \mathbb{R}^{m}$, $z_{1}, z_{1}^{\prime}, z_{2}, z_{2}^{\prime} \in \mathbb{R}^{m \times d}$,

$$
\begin{aligned}
& \left|h\left(t, x_{1}^{\prime}, y_{1}^{\prime}, z_{1}^{\prime}, x_{1}, y_{1}, z_{1}\right)-h\left(t, x_{2}^{\prime}, y_{2}^{\prime}, z_{2}^{\prime}, x_{2}, y_{2}, z_{2}\right)\right| \\
& \leq \tilde{L}_{1}\left|x_{1}^{\prime}-x_{2}^{\prime}\right|+\tilde{L}_{2}\left|y_{1}^{\prime}-y_{2}^{\prime}\right|+\tilde{L}_{3}\left|z_{1}^{\prime}-z_{2}^{\prime}\right|+L\left(\left|x_{1}-x_{2}\right|+\left|y_{1}-y_{2}\right|+\left|z_{1}-z_{2}\right|\right),
\end{aligned}
$$

where $h=b, \sigma, f$, respectively, and $b\left(\cdot, \lambda^{\prime}, \lambda\right) \in \mathcal{H}_{\mathbb{\mathbb { F }}}^{2}\left(0, T ; \mathbb{R}^{n}\right), \sigma\left(\cdot, \lambda^{\prime}, \lambda\right) \in \mathcal{H}_{\mathbb{\mathbb { F }}}^{2}\left(0, T ; \mathbb{R}^{n \times d}\right), f\left(\cdot, \lambda^{\prime}, \lambda\right) \in$ $\mathcal{H}_{\mathbb{\mathbb { F }}}^{2}\left(0, T ; \mathbb{R}^{m}\right), \lambda=(x, y, z), \lambda^{\prime}=\left(x^{\prime}, y^{\prime}, z^{\prime}\right)$

(ii) For $\left(x_{i}^{\prime}, x_{i}\right) \in \mathbb{R}^{n} \times \mathbb{R}^{n}, i=1,2$,

$$
\left|\Phi\left(x_{1}^{\prime}, x_{1}\right)-\Phi\left(x_{2}^{\prime}, x_{2}\right)\right| \leq \tilde{L}_{\Phi}\left|x_{1}^{\prime}-x_{2}^{\prime}\right|+L_{\Phi}\left|x_{1}-x_{2}\right|,
$$

and for any $x^{\prime}, x \in \mathbb{R}^{n}, \Phi\left(x^{\prime}, x\right) \in L^{2}\left(\Omega, \overline{\mathcal{F}}_{T}, \mathbb{R}^{m}\right)$.

Given an $m \times n$ full-rank matrix $G$, we put

$$
\lambda=\left(\begin{array}{l}
x \\
y \\
z
\end{array}\right), \quad \lambda^{\prime}=\left(\begin{array}{l}
x^{\prime} \\
y^{\prime} \\
z^{\prime}
\end{array}\right), \quad B\left(t, \lambda^{\prime}, \lambda\right)=\left(\begin{array}{c}
-G^{T} f \\
G b \\
G \sigma
\end{array}\right)\left(t, \lambda^{\prime}, \lambda\right) .
$$

From (H2.1) we know that $B\left(t, \lambda^{\prime}, \lambda\right)$ is Lipschitz in $\lambda^{\prime}$ and $\lambda$, uniformly with respect to $t$. Let us denote the two Lipschitz constants by $\tilde{L}_{B}$ and $L_{B}$, respectively.

(H2.2) (i) $<B\left(t, \lambda^{\prime}, \lambda\right)-B\left(t, \lambda^{\prime}, \bar{\lambda}\right), \lambda-\bar{\lambda}>\leq-\beta_{1}|\hat{x}|^{2}$

(ii) $<\Phi\left(x^{\prime}, x\right)-\Phi\left(x^{\prime}, \bar{x}\right), G(x-\bar{x})>\geq \mu_{1}|\hat{x}|^{2}$,

where $\lambda=(x, y, z), \lambda^{\prime}=\left(x^{\prime}, y^{\prime}, z^{\prime}\right), \bar{\lambda}=(\bar{x}, \bar{y}, \bar{z}), \hat{x}=x-\bar{x}, \hat{y}=y-\bar{y}, \hat{z}=z-\bar{z}$, and $\beta_{1}, \mu_{1}$ are nonnegative constants with $\beta_{1}>\tilde{L}_{B}\left(1+2 \mathrm{e}^{\hat{\beta} T}\right) ; \quad \mu_{1}>\tilde{L}_{\Phi} \lambda_{1}+4\left(\tilde{L}_{\Phi}^{2}+L_{\Phi}^{2}\right) \tilde{L}_{B} \mathrm{e}^{\hat{\beta} T}$, where $\hat{\beta}=6 L^{2}+4 \tilde{L}_{3}^{2}+2 \tilde{L}_{1}^{2}+2 \tilde{L}_{2}+2 L+1$ (in particular, as $h=b, \sigma, f$ is independent of $\left.\left(y^{\prime}, z^{\prime}\right), \hat{\beta}=4 L^{2}+2 \tilde{L}_{1}^{2}+2 L+1\right)$, and $\lambda_{1}>0$ satisfies $|G l| \leq \lambda_{1}|l|$, for any vector $l \in \mathbb{R}^{n}$.

For $a \in \mathbb{R}^{n}$, we consider the following fully coupled mean-field FBSDE:

$$
\left\{\begin{array}{l}
X_{t}=a+\int_{0}^{t} E^{\prime}\left[b\left(s, X_{s}^{\prime}, Y_{s}^{\prime}, Z_{s}^{\prime}, X_{s}, Y_{s}, Z_{s}\right)\right] \mathrm{d} s+\int_{0}^{t} E^{\prime}\left[\sigma\left(s, X_{s}^{\prime}, Y_{s}^{\prime}, X_{s}, Y_{s}\right)\right] \mathrm{d} B_{s}, \\
Y_{t}=E^{\prime}\left[\Phi\left(\left(X_{T}\right)^{\prime}, X_{T}\right)\right]+\int_{t}^{T} E^{\prime}\left[f\left(s, X_{s}^{\prime}, Y_{s}^{\prime}, Z_{s}^{\prime}, X_{s}, Y_{s}, Z_{s}\right)\right] \mathrm{d} s-\int_{t}^{T} Z_{s} \mathrm{~d} B_{s}, t \in[0, T] .
\end{array}\right.
$$

In [12], the authors proved that the fully coupled mean-field FBSDE (2.1) has a unique solution. Precisely speaking, we have:

Lemma 2.1. Under the assumptions (H2.1) and (H2.2), the fully coupled mean-field FBSDE (2.1) admits a unique solution $(X, Y, Z) \in \mathcal{S}_{\mathbb{F}}^{2}\left(0, T ; \mathbb{R}^{n}\right) \times \mathcal{S}_{\mathbb{F}}^{2}\left(0, T ; \mathbb{R}^{m}\right) \times \mathcal{H}_{\mathbb{F}}^{2}\left(0, T ; \mathbb{R}^{m \times d}\right)$.

Moreover, for any initial time $t \in[0, T]$, initial value $\zeta \in L^{2}\left(\Omega, \mathcal{F}_{t}, P ; \mathbb{R}^{n}\right)$ and given $x_{0} \in \mathbb{R}^{n}$, also the following fully coupled FBSDE:

$$
\left\{\begin{aligned}
X_{s}^{t, \zeta}= & \zeta+\int_{t}^{s} E^{\prime}\left[b\left(r,\left(X_{r}^{0, x_{0}}\right)^{\prime},\left(Y_{r}^{0, x_{0}}\right)^{\prime},\left(Z_{r}^{0, x_{0}}\right)^{\prime}, X_{r}^{t, \zeta}, Y_{r}^{t, \zeta}, Z_{r}^{t, \zeta}\right)\right] \mathrm{d} r \\
& +\int_{t}^{s} E^{\prime}\left[\sigma\left(r,\left(X_{r}^{0, x_{0}}\right)^{\prime},\left(Y_{r}^{0, x_{0}}\right)^{\prime}, X_{r}^{t, \zeta}, Y_{r}^{t, \zeta}\right)\right] \mathrm{d} B_{r}, \\
Y_{s}^{t, \zeta}= & E^{\prime}\left[\Phi\left(\left(X_{T}^{0, x_{0}}\right)^{\prime}, X_{T}^{t, \zeta}\right)\right]+\int_{s}^{T} E^{\prime}\left[f\left(r,\left(X_{r}^{0, x_{0}}\right)^{\prime},\left(Y_{r}^{0, x_{0}}\right)^{\prime},\left(Z_{r}^{0, x_{0}}\right)^{\prime}, X_{r}^{t, \zeta}, Y_{r}^{t, \zeta}, Z_{r}^{t, \zeta}\right)\right] \mathrm{d} r \\
& -\int_{s}^{T} Z_{r}^{t, \zeta} \mathrm{d} B_{r}, s \in[t, T],
\end{aligned}\right.
$$


has a unique adapted solution, denoted by $\left(X_{s}^{t, \zeta}, Y_{s}^{t, \zeta}, Z_{s}^{t, \zeta}\right)_{s \in[t, T]} \in \mathcal{S}_{\mathbb{F}}^{2}\left(t, T ; \mathbb{R}^{n}\right) \times \mathcal{S}_{\mathbb{F}}^{2}\left(t, T ; \mathbb{R}^{m}\right) \times \mathcal{H}_{\mathbb{F}}^{2}\left(t, T ; \mathbb{R}^{m \times d}\right)$. In fact, if $(t, \zeta)=\left(0, x_{0}\right)$, then $(2.2)$ changes to a fully coupled mean-field FBSDE. Thanks to Lemma 2.1, we see that this equation has a unique adapted solution, denoted by $\left(X_{s}^{0, x_{0}}, Y_{s}^{0, x_{0}}, Z_{s}^{0, x_{0}}\right)_{s \in[0, T]} \in \mathcal{S}_{\mathbb{F}}^{2}\left(0, T ; \mathbb{R}^{n}\right) \times$ $\mathcal{S}_{\mathbb{F}}^{2}\left(0, T ; \mathbb{R}^{m}\right) \times \mathcal{H}_{\mathbb{F}}^{2}\left(0, T ; \mathbb{R}^{m \times d}\right)$. Then, it can be checked that the coefficient $h^{0}(s, x, y, z) \triangleq E^{\prime}\left[h\left(s,\left(X_{s}^{0, x_{0}}\right)^{\prime}\right.\right.$, $\left.\left.\left(Y_{s}^{0, x_{0}}\right)^{\prime},\left(Z_{s}^{0, x_{0}}\right)^{\prime}, x, y, z\right)\right]$ satisfies the assumptions for classical fully coupled FBSDEs, where $h=b, \sigma, f$, respectively. Consequently, according to the existence and uniqueness theorem of classical fully coupled FBSDEs, the above equation (2.2) admits a unique solution $\left(X_{s}^{t, \zeta}, Y_{s}^{t, \zeta}, Z_{s}^{t, \zeta}\right)_{s \in[t, T]} \in \mathcal{S}_{\mathbb{F}}^{2}\left(t, T ; \mathbb{R}^{n}\right) \times \mathcal{S}_{\mathbb{F}}^{2}\left(t, T ; \mathbb{R}^{m}\right) \times$ $\mathcal{H}_{\mathbb{F}}^{2}\left(t, T ; \mathbb{R}^{m \times d}\right)$ (see, e.g., Thm. 2.2 in [17] or Thm. 3.1 in $[10]$ ).

From standard estimates for classical fully coupled FBSDEs, we have (see, e.g., Prop. 6.1 in [13]) the following:

Lemma 2.2. Under the assumptions (H2.1) and (H2.2), there exists a constant $C>0$ such that, for all $t \in[0, T]$ and $\zeta, \zeta^{\prime} \in L^{2}\left(\Omega, \mathcal{F}_{t}, P ; \mathbb{R}^{n}\right)$, P-a.s.,

(i) $E\left[\sup _{t \leq s \leq T}\left|X_{s}^{t, \zeta}-X_{s}^{t, \zeta^{\prime}}\right|^{2}+\sup _{t \leq s \leq T}\left|Y_{s}^{t, \zeta}-Y_{s}^{t, \zeta^{\prime}}\right|^{2}+\int_{t}^{T}\left|Z_{s}^{t, \zeta}-Z_{s}^{t, \zeta^{\prime}}\right|^{2} \mathrm{~d} s \mid \mathcal{F}_{t}\right] \leq C\left|\zeta-\zeta^{\prime}\right|^{2}$;

(ii) $E\left[\sup _{t \leq s \leq T}\left|X_{s}^{t, \zeta}\right|^{2}+\sup _{t \leq s \leq T}\left|Y_{s}^{t, \zeta}\right|^{2}+\int_{t}^{T}\left|Z_{s}^{t, \zeta}\right|^{2} \mathrm{~d} s \mid \mathcal{F}_{t}\right] \leq C\left(1+|\zeta|^{2}\right)$.

In particular,

(iii) $\left|Y_{t}^{t, \zeta}\right| \leq C(1+|\zeta|) ; \quad$ (iv) $\left|Y_{t}^{t, \zeta}-Y_{t}^{t, \zeta^{\prime}}\right| \leq C\left|\zeta-\zeta^{\prime}\right|$.

\section{Fully COUPled FBSDEs involving the value Function}

Let $V$ be a compact metric space. An $\mathbb{F}$-progressively measurable process $v \in\left\{v_{r}, r \in[t, s]\right\}$ taking its values in $V$ is called an admissible control process on the time interval $[t, s](0 \leq t<s \leq T)$. By $\mathcal{V}_{t, s}$ we denote the set of all admissible controls over $[t, s]$.

In this section, we study the following fully coupled FBSDE involving the value function. For $x_{0} \in \mathbb{R}^{n}$ and $\bar{v} \in \mathcal{V}_{0, T}$ arbitrarily chosen but fixed, we consider

$$
\left\{\begin{aligned}
\mathrm{d} X_{s}^{t, x ; v}= & E^{\prime}\left[b\left(s,\left(X_{s}^{0, x_{0} ; \bar{v}}\right)^{\prime}, W\left(s,\left(X_{s}^{0, x_{0} ; \bar{v}}\right)^{\prime}\right), X_{s}^{t, x ; v}, Y_{s}^{t, x ; v}, Z_{s}^{t, x ; v}, v_{s}\right)\right] \mathrm{d} s \\
& +E^{\prime}\left[\sigma\left(s,\left(X_{s}^{0, x_{0} ; \bar{v}}\right)^{\prime}, W\left(s,\left(X_{s}^{0, x_{0} ; \bar{v}}\right)^{\prime}\right), X_{s}^{t, x ; v}, Y_{s}^{t, x ; v}, v_{s}\right)\right] \mathrm{d} B_{s}, \\
\mathrm{~d} Y_{s}^{t, x ; v}= & -E^{\prime}\left[f\left(s,\left(X_{s}^{0, x_{0} ; \bar{v}}\right)^{\prime}, W\left(s,\left(X_{s}^{0, x_{0} ; \bar{v}}\right)^{\prime}\right), X_{s}^{t, x ; v}, Y_{s}^{t, x ; v}, Z_{s}^{t, x ; v}, v_{s}\right)\right] \mathrm{d} s \\
& +Z_{s}^{t, x ; v} \mathrm{~d} B_{s}, s \in[t, T], \\
X_{t}^{t, x ; v}= & x, \quad Y_{T}^{t, x ; v}=E^{\prime}\left[\Phi\left(\left(X_{T}^{0, x_{0} ; \bar{v}}\right)^{\prime}, X_{T}^{t, x ; v}\right)\right], \\
W(t, x)= & \operatorname{essinf}_{v \in \mathcal{V}_{t, T}} Y_{t}^{t, x ; v}, \quad(t, x) \in[0, T] \times \mathbb{R}^{n} .
\end{aligned}\right.
$$

Let us introduce now the assumptions on the coefficients which we need. We suppose that the functions

$$
\begin{aligned}
& b\left(t, x^{\prime}, y^{\prime}, x, y, z, v\right):[0, T] \times \mathbb{R}^{n} \times \mathbb{R} \times \mathbb{R}^{n} \times \mathbb{R} \times \mathbb{R}^{d} \times V \rightarrow \mathbb{R}^{n} \\
& \sigma\left(t, x^{\prime}, y^{\prime}, x, y, v\right):[0, T] \times \mathbb{R}^{n} \times \mathbb{R} \times \mathbb{R}^{n} \times \mathbb{R} \times V \rightarrow \mathbb{R}^{n \times d} ; \\
& f\left(t, x^{\prime}, y^{\prime}, x, y, z, v\right):[0, T] \times \mathbb{R}^{n} \times \mathbb{R} \times \mathbb{R}^{n} \times \mathbb{R} \times \mathbb{R}^{d} \times V \rightarrow \mathbb{R} \\
& \Phi\left(x^{\prime}, x\right): \mathbb{R}^{n} \times \mathbb{R}^{n} \rightarrow \mathbb{R}
\end{aligned}
$$

satisfy:

(H3.1) (i) There exist $\tilde{L}, L>0$ and $\alpha>0$ small enough (a precision for $\alpha$ will be given in Rem. 3.8) such that, for all $t \in[0, T], x_{1}, x_{1}^{\prime}, x_{2}, x_{2}^{\prime} \in \mathbb{R}^{n}, y_{1}, y_{1}^{\prime}, y_{2}, y_{2}^{\prime} \in \mathbb{R}, z_{1}, z_{2} \in \mathbb{R}^{d}, v \in V$,

$$
\begin{aligned}
& \left|h\left(t, x_{1}^{\prime}, y_{1}^{\prime}, x_{1}, y_{1}, z_{1}, v\right)-h\left(t, x_{2}^{\prime}, y_{2}^{\prime}, x_{2}, y_{2}, z_{2}, v\right)\right| \\
& \leq \tilde{L}\left|x_{1}^{\prime}-x_{2}^{\prime}\right|+\alpha\left|y_{1}^{\prime}-y_{2}^{\prime}\right|+L\left(\left|x_{1}-x_{2}\right|+\left|y_{1}-y_{2}\right|+\left|z_{1}-z_{2}\right|\right),
\end{aligned}
$$

where $h=b, \sigma, f$; 
(ii) For $h=b, \sigma, f, h\left(t, x^{\prime}, y^{\prime}, x, y, z, v\right)$ is continuous with respect to $(t, v)$, uniformly in $\left(x^{\prime}, y^{\prime}, x, y, z\right)$;

(iii) $\Phi\left(x^{\prime}, x\right)$ is uniformly Lipschitz in $\left(x^{\prime}, x\right)$, i.e., there exist $\tilde{L}_{\Phi}, L_{\Phi}>0$ such that, for $x_{1}, x_{1}^{\prime}, x_{2}, x_{2}^{\prime} \in \mathbb{R}^{n}$,

$$
\left|\Phi\left(x_{1}^{\prime}, x_{1}\right)-\Phi\left(x_{2}^{\prime}, x_{2}\right)\right| \leq \tilde{L}_{\Phi}\left|x_{1}^{\prime}-x_{2}^{\prime}\right|+L_{\Phi}\left|x_{1}-x_{2}\right| .
$$

Further assumptions on the coefficients are made through the function

$$
B\left(t, \lambda^{\prime}, \lambda, v\right)=\left(\begin{array}{c}
-G^{T} f \\
G b \\
G \sigma
\end{array}\right)\left(t, \lambda^{\prime}, \lambda, v\right), \quad t \in[0, T], v \in V, \lambda=\left(\begin{array}{c}
x \\
y \\
z
\end{array}\right), \lambda^{\prime}=\left(\begin{array}{c}
x^{\prime} \\
y^{\prime}
\end{array}\right)
$$

where $G^{T} \in \mathbb{R}^{n} \backslash\{0\},\left|G^{T}\right|=1$ is suitably chosen.

As indicated in Section 2, under the assumption (H3.1)-(i), $B\left(t, \lambda^{\prime}, \lambda, v\right)$ is Lipschitz in $\lambda^{\prime}$ and $\lambda$. By $\tilde{L}_{B}$ and $L_{B}$ we denote their respective Lipschitz constants. From Hölder inequality, we have

$$
\left|B\left(t, \overline{\lambda^{\prime}}, \bar{\lambda}, v\right)-B\left(t, \lambda^{\prime}, \lambda, v\right)\right| \leq \sqrt{3} \sqrt{\tilde{L}^{2}+\alpha^{2}}\left|\widehat{\lambda^{\prime}}\right|+3 L|\widehat{\lambda}|,
$$

where $\widehat{\lambda^{\prime}}=\overline{\lambda^{\prime}}-\lambda^{\prime}, \hat{\lambda}=\bar{\lambda}-\lambda$. Hence here we can set $\tilde{L}_{B}=\sqrt{3} \sqrt{\tilde{L}^{2}+\alpha^{2}}$ and $L_{B}=3 L$.

We suppose the following monotonicity conditions

(H3.2)] (i) $<B\left(t, \lambda^{\prime}, \lambda, v\right)-B\left(t, \lambda^{\prime}, \bar{\lambda}, v\right), \lambda-\bar{\lambda}>\leq-\beta_{1}|\hat{x}|^{2}$;

(ii) $<G^{T}\left(\Phi\left(x^{\prime}, x\right)-\Phi\left(x^{\prime}, \bar{x}\right)\right), x-\bar{x}>\geq \mu_{1}|\hat{x}|^{2}$,

where $\lambda=(x, y, z), \bar{\lambda}=(\bar{x}, \bar{y}, \bar{z}), \lambda^{\prime}=\left(x^{\prime}, y^{\prime}, z^{\prime}\right), \hat{x}=x-\bar{x}, \hat{y}=y-\bar{y}, \hat{z}=z-\bar{z}, x, \bar{x}, x^{\prime} \in \mathbb{R}^{n}, y, \bar{y}, y^{\prime} \in$ $\mathbb{R}, z, \bar{z}, z^{\prime} \in \mathbb{R}^{d}, \beta_{1} \geq 0, \mu_{1} \geq 0$, with $\beta_{1}>\tilde{L}_{B}\left(1+2 \mathrm{e}^{\bar{\beta} T}\right), \quad \mu_{1}>\tilde{L}_{\Phi}+4 \tilde{L}_{B}\left(\tilde{L}_{\Phi}^{2}+L_{\Phi}^{2}\right) \mathrm{e}^{\bar{\beta} T}$.

Here $\bar{\beta}=4 L^{2}+2 \tilde{L}^{2}+2 L+2 \alpha+1$, and $L, \tilde{L}$ and $\alpha$ are the Lipschitz constants of $h=b, \sigma, f$ introduced in (H3.1)-(i).

Moreover, we need the following assumption

(H3.3) $h\left(t, x^{\prime}, y^{\prime}, 0,0,0, v\right)$ and $\Phi\left(x^{\prime}, 0\right)$ are bounded, i.e., there is a constant $L_{b}>0$ such that, for all $t \in$ $[0, T], x^{\prime} \in \mathbb{R}^{n}, y^{\prime} \in \mathbb{R}, v \in V,\left|\Phi\left(x^{\prime}, 0\right)\right| \leq L_{b} ; \quad\left|h\left(t, x^{\prime}, y^{\prime}, 0,0,0, v\right)\right| \leq L_{b}$, for $h=b, \sigma, f$, respectively.

Remark 3.1. Under the assumptions (H3.1) and (H3.3), $h=b, \sigma, f$ and $\Phi$ are of linear growth with respect to $(x, y, z)$ and $x$, uniformly in $\left(t, x^{\prime}, y^{\prime}, v\right)$ and $x^{\prime}$, respectively: For $t \in[0, T], x^{\prime}, x \in \mathbb{R}^{n}, y^{\prime}, y \in \mathbb{R}, z \in \mathbb{R}^{d}, v \in V$, $\left|\Phi\left(x^{\prime}, x\right)\right| \leq\left(L_{\Phi}+L_{b}\right)(1+|x|) ; \quad\left|h\left(t, x^{\prime}, y^{\prime}, x, y, z, v\right)\right| \leq\left(L+L_{b}\right)(1+|x|+|y|+|z|)$.

Let $K>0$ be a given constant and $\tilde{W}:[0, T] \times \mathbb{R}^{n} \rightarrow \mathbb{R}$ be a deterministic Borel function such that, $|\tilde{W}(t, x)| \leq K(1+|x|)$, and $|\tilde{W}(t, x)-\tilde{W}(t, \bar{x})| \leq K|x-\bar{x}|$, for all $t \in[0, T], x, \bar{x} \in \mathbb{R}^{n}$. For arbitrarily chosen but fixed $x_{0} \in \mathbb{R}^{n}, \bar{v} \in \mathcal{V}_{0, T}$, and for $(t, x) \in[0, T] \times \mathbb{R}^{n}, v \in \mathcal{V}_{t, T}$, let us consider the following fully coupled FBSDE:

$$
\left\{\begin{aligned}
\mathrm{d} X_{s}^{t, x ; v}= & E^{\prime}\left[b\left(s,\left(X_{s}^{0, x_{0} ; \bar{v}}\right)^{\prime}, \tilde{W}\left(s,\left(X_{s}^{0, x_{0} ; \bar{v}}\right)^{\prime}\right), X_{s}^{t, x ; v}, Y_{s}^{t, x ; v}, Z_{s}^{t, x ; v}, v_{s}\right)\right] \mathrm{d} s \\
& +E^{\prime}\left[\sigma\left(s,\left(X_{s}^{0, x_{0} ; \bar{v}}\right)^{\prime}, \tilde{W}\left(s,\left(X_{s}^{0, x_{0} ; \bar{v}}\right)^{\prime}\right), X_{s}^{t, x ; v}, Y_{s}^{t, x ; v}, v_{s}\right)\right] \mathrm{d} B_{s}, \\
\mathrm{~d} Y_{s}^{t, x ; v}= & -E^{\prime}\left[f\left(s,\left(X_{s}^{0, x_{0} ; \bar{v}}\right)^{\prime}, \tilde{W}\left(s,\left(X_{s}^{0, x_{0} ; \bar{v}}\right)^{\prime}\right), X_{s}^{t, x ; v}, Y_{s}^{t, x ; v}, Z_{s}^{t, x ; v}, v_{s}\right)\right] \mathrm{d} s \\
& +Z_{s}^{t, x ; v} \mathrm{~d} B_{s}, \\
X_{t}^{t, x ; v}= & x, \quad Y_{T}^{t, x ; v}=E^{\prime}\left[\Phi\left(\left(X_{T}^{0, x_{0} ; \bar{v}}\right)^{\prime}, X_{T}^{t, x ; v}\right)\right] .
\end{aligned}\right.
$$

Lemma 3.2. We suppose that the assumptions (H3.1)-(H3.3) hold true. Then the fully coupled FBSDE (3.2) admits a unique solution $\left(X_{s}^{t, x ; v}, Y_{s}^{t, x ; v}, Z_{s}^{t, x ; v}\right)_{s \in[t, T]} \in \mathcal{S}_{\mathbb{F}}^{2}\left(t, T ; \mathbb{R}^{n}\right) \times \mathcal{S}_{\mathbb{F}}^{2}(t, T ; \mathbb{R}) \times \mathcal{H}_{\mathbb{F}}^{2}\left(t, T ; \mathbb{R}^{d}\right)$. 
Proof. For $\left(s, x^{\prime}, x, y, z\right) \in[0, T] \times \mathbb{R}^{n} \times \mathbb{R}^{n} \times \mathbb{R} \times \mathbb{R}^{d}$, we define $\tilde{h}\left(s, x^{\prime}, x, y, z\right) \triangleq h\left(s, x^{\prime}, \tilde{W}\left(s, x^{\prime}\right), x, y, z, v_{s}\right)$, $h=b, \sigma, f$, respectively. Then, for $s \in[0, T], x_{1}^{\prime}, x_{2}^{\prime}, x_{1}, x_{2} \in \mathbb{R}^{n}, y_{1}, y_{2} \in \mathbb{R}, z_{1}, z_{2} \in \mathbb{R}^{d}$,

$$
\left|\tilde{h}\left(s, x_{1}^{\prime}, x_{1}, y_{1}, z_{1}\right)-\tilde{h}\left(s, x_{2}^{\prime}, x_{2}, y_{2}, z_{2}\right)\right| \leq(\tilde{L}+\alpha K)\left|x_{1}^{\prime}-x_{2}^{\prime}\right|+L\left(\left|x_{1}-x_{2}\right|+\left|y_{1}-y_{2}\right|+\left|z_{1}-z_{2}\right|\right),
$$

where $L, \tilde{L}$ and $\alpha$ are the Lipschitz constants given in (H3.1)-(i) and $K$ is the Lipschitz and the linear growth constant of $\tilde{W}$.

We put

$$
\tilde{B}\left(s, \lambda^{\prime}, \lambda\right) \triangleq\left(\begin{array}{c}
-G^{T} \tilde{f} \\
G \tilde{b} \\
G \tilde{\sigma}
\end{array}\right)\left(s, \lambda^{\prime}, \lambda\right), \quad \tilde{\Phi}\left(x^{\prime}, x\right) \triangleq \Phi\left(x^{\prime}, x\right),
$$

where $\lambda=(x, y, z), \lambda^{\prime}=\left(x^{\prime}, y^{\prime}, z^{\prime}\right)$.

By $\tilde{L}_{\tilde{B}}, L_{\tilde{B}}$ and $\tilde{L}_{\tilde{\Phi}}, L_{\tilde{\Phi}}$ we denote the Lipschitz constants of $\tilde{B}$ and $\tilde{\Phi}$ with respect to $\lambda^{\prime}, \lambda$ and $x^{\prime}, x$, respectively. From Hölder's inequality we can obtain

$$
\left|\tilde{B}\left(s, \overline{\lambda^{\prime}}, \bar{\lambda}\right)-\tilde{B}\left(s, \lambda^{\prime}, \lambda\right)\right| \leq \sqrt{3}(\tilde{L}+\alpha K)\left|\widehat{\lambda^{\prime}}\right|+3 L|\hat{\lambda}|,
$$

where $\hat{k}=\bar{k}-k, k=\lambda^{\prime}, \lambda$. Consequently, we can take $\tilde{L}_{\tilde{B}}=\sqrt{3}(\tilde{L}+\alpha K)$ and $L_{\tilde{B}}=3 L$. Notice that $\alpha$ is small enough such that $\beta_{1}>\tilde{L}_{\tilde{B}}\left(1+2 \mathrm{e}^{\tilde{\beta} T}\right), \quad \mu_{1}>\tilde{L}_{\tilde{\Phi}}+4 \tilde{L}_{\tilde{B}}\left(\tilde{L}_{\tilde{\Phi}}^{2}+L_{\tilde{\Phi}}^{2}\right) \mathrm{e}^{\tilde{\beta} T}$, where $\tilde{\beta}=4 L^{2}+2(\tilde{L}+\alpha K)^{2}+2 L+1$, $\tilde{L}_{\tilde{\Phi}}=\tilde{L}_{\Phi}, L_{\tilde{\Phi}}=L_{\Phi}$ (see Rem. 3.8). According to Lemma 2.1, for $(t, x)=\left(0, x_{0}\right)$ and given $\bar{v} \in \mathcal{V}_{0, T}$, the fully coupled FBSDE (3.2) with coefficients $\tilde{b}, \tilde{\sigma}, \tilde{f}, \tilde{\Phi}$ admits a unique solution, denoted by $\left(X_{s}^{0}, Y_{s}^{0}, Z_{s}^{0}\right)_{s \in[0, T]} \in$ $\mathcal{S}_{\mathbb{F}}^{2}\left(0, T ; \mathbb{R}^{n}\right) \times \mathcal{S}_{\mathbb{F}}^{2}(0, T ; \mathbb{R}) \times \mathcal{H}_{\mathbb{F}}^{2}\left(0, T ; \mathbb{R}^{d}\right)$.

Hence we can define

$$
h^{0}(s, x, y, z) \triangleq E^{\prime}\left[h\left(s,\left(X_{s}^{0}\right)^{\prime}, \tilde{W}\left(s,\left(X_{s}^{0}\right)^{\prime}\right), x, y, z, v_{s}\right)\right] ; \quad \Phi^{0}(x) \triangleq E^{\prime}\left[\Phi\left(\left(X_{T}^{0}\right)^{\prime}, x\right)\right],
$$

where $h=b, \sigma, f$, respectively. Obviously, $b^{0}, \sigma^{0}, f^{0}$ and $\Phi^{0}$ are Lipschitz with respect to $(x, y, z)$ and $x$ with the same Lipschitz constants as $b, \sigma, f$ and $\Phi$ in $(x, y, z)$ and $x$, respectively, and satisfy the monotonicity condition for classical fully coupled FBSDEs with the constants $\beta_{1}$ and $\mu_{1}$ which do not depend on $\tilde{W}$. Consequently, for $(t, x, v) \in[0, T] \times \mathbb{R}^{n} \times \mathcal{V}_{t, T}$, there is a unique triple $\left(X_{s}^{t, x ; v}, Y_{s}^{t, x ; v}, Z_{s}^{t, x ; v}\right)_{s \in[t, T]} \in \mathcal{S}_{\mathbb{F}}^{2}\left(t, T ; \mathbb{R}^{n}\right) \times \mathcal{S}_{\mathbb{F}}^{2}(t, T ; \mathbb{R}) \times$ $\mathcal{H}_{\mathbb{F}}^{2}\left(t, T ; \mathbb{R}^{d}\right)$ satisfying the fully coupled FBSDE (3.2) (see, Thm. 3.1 in [10] or Thm. 2.2 in [17]).

Lemma 3.3. Let us suppose that (H3.1)-(H3.3) hold true and $t \in[0, T], x, \bar{x} \in \mathbb{R}^{n}, v \in \mathcal{V}_{t, T}$. By $\left(X_{s}^{t, x ; v}, Y_{s}^{t, x ; v}, Z_{s}^{t, x ; v}\right)_{s \in[t, T]}$ (resp., $\left.\left(X_{s}^{t, \bar{x} ; v}, Y_{s}^{t, \bar{x} ; v}, Z_{s}^{t, \bar{x} ; v}\right)_{s \in[t, T]}\right)$ we denote the solution of the fully coupled $F B$ $S D E(3.2)$ associated with initial value $(t, x) \in[0, T] \times \mathbb{R}^{n}$ (resp., $(t, \bar{x}) \in[0, T] \times \mathbb{R}^{n}$ ) and $v \in \mathcal{V}_{t, T}$. Then, for any $p \geq 2$, there exists a constant $L_{0}>0$ depending on $L, L_{\Phi}, L_{b}$ and $p$ such that $P$-a.s.,

$$
\begin{aligned}
& \text { (i) } E\left[\sup _{t \leq s \leq T}\left(\left|X_{s}^{t, x ; v}-X_{s}^{t, \bar{x} ; v}\right|^{2}+\left|Y_{s}^{t, x ; v}-Y_{s}^{t, \bar{x} ; v}\right|^{2}\right)+\int_{t}^{T}\left|Z_{s}^{t, x ; v}-Z_{s}^{t, \bar{x} ; v}\right|^{2} \mathrm{~d} s \mid \mathcal{F}_{t}\right] \leq L_{0}^{2}|x-\bar{x}|^{2} ; \\
& \text { (ii) } E\left[\sup _{t \leq s \leq T}\left(\left|X_{s}^{t, x ; v}\right|^{p}+\left|Y_{s}^{t, x ; v}\right|^{p}\right)+\left(\int_{t}^{T}\left|Z_{s}^{t, x ; v}\right|^{2} \mathrm{~d} s\right)^{\frac{p}{2}} \mid \mathcal{F}_{t}\right] \leq L_{0}^{p}\left(1+|x|^{p}\right) ;
\end{aligned}
$$

and, in particular,

(iii) $\left|Y_{t}^{t, x ; v}-Y_{t}^{t, \bar{x} ; v}\right| \leq L_{0}|x-\bar{x}| ; \quad$ (iv) $\left|Y_{t}^{t, x ; v}\right| \leq L_{0}(1+|x|)$.

Proof. From the proof of Lemma 3.2, we know that the fully coupled FBSDE (3.2) can be treated as a classical fully coupled FBSDE with coefficients $b^{0}, \sigma^{0}, f^{0}$ and $\Phi^{0}$. Thanks to the assumptions (H3.1) and (H3.3), it is easy to get that $b^{0}, \sigma^{0}, f^{0}$ (resp., $\Phi^{0}$ ) have the same Lipschitz and linear growth constants in $(x, y, z)($ resp., $x)$ as $b, \sigma, f$ (resp., $\Phi$ ), and these Lipschitz and linear growth constants do not depend on $\tilde{W}$. According to standard estimates of classical fully coupled FBSDEs (see [13]), we have that there exists a constant $L_{0}$ depending only on $L, L_{\Phi}, L_{b}$ and $p$ such that (3.4) holds. 
Let us define $W(t, x) \triangleq \underset{v \in \mathcal{V}_{t, T}}{\operatorname{essinf}} Y_{t}^{t, x ; v},(t, x) \in[0, T] \times \mathbb{R}^{n}$, where $\left(X^{t, x ; v}, Y^{t, x ; v}, Z^{t, x ; v}\right)$ is the solution of the fully coupled FBSDE (3.2). Obviously, for the constant $L_{0}$ given in Lemma 3.3 , we have, for $t \in[0, T], x, \bar{x} \in \mathbb{R}^{n}$, P-a.s.,

$$
\begin{aligned}
& \text { (i) }|W(t, x)| \leq L_{0}(1+|x|) \text {; } \\
& \text { (ii) }|W(t, x)-W(t, \bar{x})| \leq L_{0}|x-\bar{x}| .
\end{aligned}
$$

This means, no matter what the Lipschitz and the linear growth constants of $\tilde{W}$ in fully coupled FBSDE (3.2) are, the value function $W(t, x)$ defined by the solution of fully coupled FBSDE (3.2) with $\tilde{W}$ always has the same Lipschitz and linear growth constant $L_{0}$ depending only on $L, L_{\Phi}$ and $L_{b}$.

Notice that due to the definition as essential infimum over a family of $\mathcal{F}_{t}$-measurable random variables, $W(t, x)$ is a priori a $\mathcal{F}_{t}$-measurable random variable. However, we can even prove that the value function $W$ is deterministic with the Girsanov transformation.

Lemma 3.4. We suppose the assumptions (H3.1)-(H3.3) hold true. The value function $W$ is deterministic and can be characterized by $E[W]$, i.e., $W(t, x)=E[W(t, x)],(t, x) \in[0, T] \times \mathbb{R}^{n}, P$-a.s.

The proof is similar to Proposition 3.1 in Li and Wei [13]. Thus we omit it.

Remark 3.5. The two inequalities for parameters $\beta_{1}, \mu_{1}$ in assumption (H3.2) can be reduced to

$$
\beta_{1}>\sqrt{3} \tilde{L}\left(1+2 \mathrm{e}^{\bar{\beta} T}\right), \quad \mu_{1}>\tilde{L}_{\Phi}+4 \sqrt{3} \tilde{L}\left(\tilde{L}_{\Phi}^{2}+L_{\Phi}^{2}\right) \mathrm{e}^{\bar{\beta} T},
$$

where $\bar{\beta}=4 L^{2}+2 \tilde{L}^{2}+2 L+1$. In fact, what we really use in the proof of Lemma 3.2 are the two inequalities:

$$
\begin{aligned}
& \beta_{1}>\sqrt{3}(\tilde{L}+\alpha K)\left(1+2 \mathrm{e}^{T\left\{4 L^{2}+2(\tilde{L}+\alpha K)^{2}+2 L+1\right\}}\right), \\
& \mu_{1}>\tilde{L}_{\Phi}+4 \sqrt{3}(\tilde{L}+\alpha K)\left(\tilde{L}_{\Phi}^{2}+L_{\Phi}^{2}\right) \mathrm{e}^{T\left\{4 L^{2}+2(\tilde{L}+\alpha K)^{2}+2 L+1\right\}} .
\end{aligned}
$$

Moreover, from Lemma 3.3, we also see that the Lipschitz and the linear growth constant $L_{0}$ of $W$ does not depend on the choice of $K$. Consequently, let $\tilde{W} \equiv 0$, i.e., $K=0$, the same constant $L_{0}$ can still be obtained. Hence, the two inequalities for the parameters $\beta_{1}, \mu_{1}$ in (H3.2) have only to satisfy (3.6).

However, in what follows, we use the following assumption instead of (H3.2).

(H3.2L $\left.\left.\mathbf{L}_{\mathbf{0}}\right)\right](\mathrm{i})<B\left(t, \lambda^{\prime}, \lambda, v\right)-B\left(t, \lambda^{\prime}, \bar{\lambda}, v\right), \lambda-\bar{\lambda}>\leq-\beta_{1}|\hat{x}|^{2}$;

(ii) $<G^{T}\left(\Phi\left(x^{\prime}, x\right)-\Phi\left(x^{\prime}, \bar{x}\right)\right), x-\bar{x}>\geq \mu_{1}|\hat{x}|^{2}$,

where $\lambda=(x, y, z), \bar{\lambda}=(\bar{x}, \bar{y}, \bar{z}), \lambda^{\prime}=\left(x^{\prime}, y^{\prime}, z^{\prime}\right), \hat{x}=x-\bar{x}, \hat{y}=y-\bar{y}, \hat{z}=z-\bar{z}, x, \bar{x}, x^{\prime} \in \mathbb{R}^{n}, y, \bar{y}, y^{\prime} \in$ $\mathbb{R}, z, \bar{z}, z^{\prime} \in \mathbb{R}^{d}, \beta_{1} \geq 0, \mu_{1} \geq 0$, with $\beta_{1}>2\left(\tilde{L}+\alpha L_{0}+\alpha\right)\left(1+2 \mathrm{e}^{\beta T}\right), \mu_{1}>\tilde{L}_{\Phi}+8\left(\tilde{L}+\alpha L_{0}+\alpha\right)\left(\tilde{L}_{\Phi}^{2}+L_{\Phi}^{2}\right) \mathrm{e}^{\beta T}$, where $\beta=4 L^{2}+2\left(\tilde{\tilde{L}}+\alpha L_{0}\right)^{2}+2 L+2 \alpha+1$, and $L, \tilde{L}$ and $\alpha$ are the Lipschitz constants of $h=b, \sigma, f$ introduced in (H3.1)-(i), and $L_{0}>0$ is given in Lemma 3.3.

Obviously, under the assumption $\left(\mathrm{H} 3.2 \mathrm{~L}_{0}\right),(3.6)$ holds true.

Let $\mathcal{W} \triangleq\left\{W:[0, T] \times \mathbb{R}^{n} \rightarrow \mathbb{R}\right.$ measurable ||$W(t, 0)\left|\leq L_{0},\right| W(t, x)-W\left(t, x^{\prime}\right)\left|\leq L_{0}\right| x-x^{\prime} \mid$, for all $t \in$ $\left.[0, T], x, x^{\prime} \in \mathbb{R}^{n}\right\}$.

Let $b, \sigma, f$ and $\Phi$ satisfy (H3.1), (H3.2 $\left.\mathrm{L}_{0}\right)$ and (H3.3). From Lemma 3.2 we know that, for any $\tilde{W} \in \mathcal{W}$, the fully coupled FBSDE (3.2) has a unique adapted solution $\left(X_{s}^{t, x ; v}, Y_{s}^{t, x ; v}, Z_{s}^{t, x ; v}\right)_{s \in[t, T]} \in \mathcal{S}_{\mathbb{F}}^{2}\left(t, T ; \mathbb{R}^{n}\right) \times \mathcal{S}_{\mathbb{F}}^{2}(t, T ; \mathbb{R}) \times$ $\mathcal{H}_{\mathbb{F}}^{2}\left(t, T ; \mathbb{R}^{d}\right)$. We define $\hat{W}(t, x)=\underset{v \in \mathcal{V}_{t, T}}{\operatorname{essinf}} Y_{t}^{t, x ; v},(t, x) \in[0, T] \times \mathbb{R}^{n}$. Due to Lemma $3.4, \hat{W}$ is deterministic. Moreover, from (3.4), for all $t \in[0, T], x, \bar{x} \in \mathbb{R}^{n}$,
(i) $\mid$
$|\hat{W}(t, x)-\hat{W}(t, \bar{x})| \leq L_{0}|x-\bar{x}|$
(ii) $|\hat{W}(t, x)| \leq L_{0}(1+|x|)$. 
Putting now $\psi(\tilde{W}) \triangleq \hat{W}$, we define a mapping $\psi: \mathcal{W} \rightarrow \mathcal{W}$. Let $M>0, K_{M} \triangleq[0, T] \times \overline{B_{M}(0)}$, and let us consider the following supremum norm of the restriction of $W \in \mathcal{W}$ to $K_{M}$ :

Definition 3.6. For $W \in \mathcal{W}$ we define

$$
\|W\|_{K_{M}} \triangleq \sup _{t \in[0, T],|x| \leq M}|W(t, x)| .
$$

Now we state the existence and the uniqueness of the fully coupled FBSDE (3.1) involving the value function.

Theorem 3.7. We suppose that the assumptions (H3.1), (H3.2 $\left.\mathrm{L}_{0}\right)$, (H3.3) hold true. Then the fully coupled $F B S D E$ (3.1) involving the value function has a unique solution $\left\{\left(X_{s}^{t, x ; v}, Y_{s}^{t, x ; v}, Z_{s}^{t, x ; v}\right)_{s \in[t, T]} \in \mathcal{S}_{\mathbb{F}}^{2}\left(t, T ; \mathbb{R}^{n}\right) \times\right.$ $\left.\mathcal{S}_{\mathbb{F}}^{2}(t, T ; \mathbb{R}) \times \mathcal{H}_{\mathbb{F}}^{2}\left(t, T ; \mathbb{R}^{d}\right),(t, x) \in[0, T] \times \mathbb{R}^{n}, W \in \mathcal{W}\right\}$

Proof. Let $W^{0}(t, x) \equiv 0,(t, x) \in[0, T] \times \mathbb{R}^{n}$. Obviously, $W^{0} \in \mathcal{W}$. Moreover, given $W^{i} \in \mathcal{W}, W^{i+1} \triangleq \psi\left(W^{i}\right)$ belongs again to $\mathcal{W}$. Thus, we get by iteration a sequence $\left(W^{i}\right)_{i \geq 0} \subset \mathcal{W}$.

For $(t, x, v) \in[0, T] \times \mathbb{R}^{n} \times \mathcal{V}_{t, T}$, let $\left(X^{t, x ; v, i}, Y^{t, x ; v, i}, Z^{t, x ; v, i}\right)$ be the solution of the fully coupled FBSDE (3.2) associated with $W^{i}$ :

$$
\left\{\begin{aligned}
\mathrm{d} X_{s}^{t, x ; v, i}= & E^{\prime}\left[b\left(s,\left(X_{s}^{0, x_{0} ; \bar{v}, i}\right)^{\prime}, W^{i}\left(s,\left(X_{s}^{0, x_{0} ; \bar{v}, i}\right)^{\prime}\right), X_{s}^{t, x ; v, i}, Y_{s}^{t, x ; v, i}, Z_{s}^{t, x ; v, i}, v_{s}\right)\right] \mathrm{d} s \\
& +E^{\prime}\left[\sigma\left(s,\left(X_{s}^{0, x_{0} ; \bar{v}, i}\right)^{\prime}, W^{i}\left(s,\left(X_{s}^{0, x_{0} ; \bar{v}, i}\right)^{\prime}\right), X_{s}^{t, x ; v, i}, Y_{s}^{t, x ; v, i}, v_{s}\right)\right] \mathrm{d} B_{s}, \\
\mathrm{~d} Y_{s}^{t, x ; v, i}= & -E^{\prime}\left[f\left(s,\left(X_{s}^{0, x_{0} ; \bar{v}, i}\right)^{\prime}, W^{i}\left(s,\left(X_{s}^{0, x_{0} ; \bar{v}, i}\right)^{\prime}\right), X_{s}^{t, x ; v, i}, Y_{s}^{t, x ; v, i}, Z_{s}^{t, x ; v, i}, v_{s}\right)\right] \mathrm{d} s \\
& +Z_{s}^{t, x ; v, i} \mathrm{~d} B_{s}, \\
X_{t}^{t, x ; v, i}= & x, \quad Y_{T}^{t, x ; v, i}=E^{\prime}\left[\Phi\left(\left(X_{T}^{0, x_{0} ; \bar{v}, i}\right)^{\prime}, X_{T}^{t, x ; v, i}\right)\right] .
\end{aligned}\right.
$$

In the particular case $(t, x, v)=\left(0, x_{0}, \bar{v}\right)$, we write $\left(X^{0, x_{0} ; \bar{v}, i}, Y^{0, x_{0} ; \bar{v}, i}, Z^{0, x_{0} ; \bar{v}, i}\right)$ instead of $\left(X^{t, x ; v, i}, Y^{t, x ; v, i}\right.$, $\left.Z^{t, x ; v, i}\right)$.

For any $(t, x) \in[0, T] \times \mathbb{R}^{n}$ and $v \in \mathcal{V}_{t, T}$, we put, $\hat{X}^{t, x ; v, i} \triangleq X^{t, x ; v, i+1}-X^{t, x ; v, i}, \quad \hat{Y}^{t, x ; v, i} \triangleq Y^{t, x ; v, i+1}-$ $Y^{t, x ; v, i}, \quad \hat{Z}^{t, x ; v, i} \triangleq Z^{t, x ; v, i+1}-Z^{t, x ; v, i}, \quad \hat{W}^{i}(t, x) \triangleq W^{i+1}(t, x)-W^{i}(t, x), i \geq 0$. In particular, $\hat{X}^{0, x_{0} ; \bar{v}^{, i} \triangleq}$ $X^{0, x_{0} ; \bar{v}, i+1}-X^{0, x_{0} ; \bar{v}, i}, \hat{Y}^{0, x_{0} ; \bar{v}, i} \triangleq Y^{0, x_{0} ; \bar{v}, i+1}-Y^{0, x_{0} ; \bar{v}, i}, \quad \hat{Z}^{0, x_{0} ; \bar{v}, i} \triangleq Z^{0, x_{0} ; \bar{v}, i+1}-Z^{0, x_{0} ; \bar{v}, i}$.

We split the proof into the following two steps.

Step 1. We prove that the sequence $\left(X^{t, x ; v, i}, Y^{t, x ; v, i}, Z^{t, x ; v, i}, W^{i}\right)$ converge in $\mathcal{S}_{\mathbb{F}}^{2}\left(t, T ; \mathbb{R}^{n}\right) \times \mathcal{S}_{\mathbb{F}}^{2}(t, T ; \mathbb{R})$ $\times \mathcal{H}_{\mathbb{F}}^{2}\left(t, T ; \mathbb{R}^{d}\right) \times \mathcal{W}$ to some quadruple $\left(X^{t, x ; v}, Y^{t, x ; v}, Z^{t, x ; v}, W\right)$, such that $\left(X^{t, x ; v}, Y^{t, x ; v}, Z^{t, x ; v}\right)$ is the unique solution of the fully coupled FBSDE (3.2) associated with $W$ instead of $\tilde{W}$.

We prove the existence of the solution with the help of an iteration method.

From Lemma A.3, we know that there exists a constant $C_{0}>0$ independent of $(t, x) \in[0, T] \times \mathbb{R}^{n}$ (Indeed, $C_{0}$ depends only on $\tilde{L}, L, L_{0}$ and $\left.L_{\Phi}, \tilde{L}_{\Phi}, \alpha\right)$ such that

$$
E\left[\left|Y_{t}^{t, x ; v, i+1}-Y_{t}^{t, x ; v, i}\right|^{2}\right] \leq \alpha C_{0}\left(\left\|\hat{W}^{i}\right\|_{K_{M}}^{2}+\varepsilon\right) .
$$

From the Girsanov transformation argument (see Prop. 3.3 in [4]), we have

$$
W^{i+1}(t, x)=\operatorname{essinf}_{v \in \mathcal{V}_{t, T}} Y_{t}^{t, x ; v, i}=\inf _{v \in \mathcal{V}_{t, T}} E\left[Y_{t}^{t, x ; v, i}\right], \quad(t, x) \in[0, T] \times \mathbb{R}^{n}, i \geq 0 .
$$

Hence, for $(t, x) \in[0, T] \times \mathbb{R}^{n}$, using that $\hat{W}^{i+1}(t, x)=W^{i+2}(t, x)-W^{i+1}(t, x)$, we get, by Lemma A.3

$$
\left|\hat{W}^{i+1}(t, x)\right|^{2}=\left|W^{i+2}(t, x)-W^{i+1}(t, x)\right|^{2} \leq \sup _{v \in \mathcal{V}_{t, T}} E\left|\hat{Y}_{t}^{t, x ; v, i}\right|^{2} \leq \alpha C_{0}\left(\left\|\hat{W}^{i}\right\|_{K_{M}}^{2}+\varepsilon\right),
$$

where $C_{0}$ depends only on $\tilde{L}, L, L_{0}$ and $L_{\Phi}, \tilde{L}_{\Phi}, \alpha$. Therefore, for all $i \geq 0$,

$$
\left\|\hat{W}^{i+1}\right\|_{\infty}^{2} \leq \alpha C_{0}\left(\left\|\hat{W}^{i}\right\|_{K_{M}}^{2}+\varepsilon\right) \leq \alpha C_{0}\left(\left\|\hat{W}^{i}\right\|_{\infty}^{2}+\varepsilon\right)
$$


Letting $\varepsilon \downarrow 0$, we get

$$
\left\|\hat{W}^{i+1}\right\|_{\infty}^{2} \leq \alpha C_{0}\left\|\hat{W}^{i}\right\|_{\infty}^{2}, i \geq 0 .
$$

Notice that $\alpha$ is small sufficiently such that $\alpha C_{0}<1$ (see Rem. 3.8). Consequently, there exists a deterministic $W:[0, T] \times \mathbb{R}^{n} \rightarrow \mathbb{R}$ such that $\left\|W^{i}-W\right\|_{\infty} \rightarrow 0$, as $i \rightarrow \infty$. Moreover, as $\left(W^{i}\right)_{i \geq 0} \subset \mathcal{W}$, also $W \in \mathcal{W}$.

Using the argument of Lemmas A.1-A.3 again, but now with $E \int_{0}^{T}\left|\hat{W}^{i}\left(s, X_{s}^{0, x_{0} ; \bar{v}, i}\right)\right|^{2} \mathrm{~d} s \leq T|| \hat{W}^{i} \|_{\infty}^{2}$ instead of $E \int_{0}^{T}\left|\hat{W}^{i}\left(s, X_{s}^{0, x_{0} ; \bar{v}, i}\right)\right|^{2} \mathrm{~d} s \leq T\left\|\hat{W}^{i}\right\|_{K_{M}}^{2}+\varepsilon$, we get, for all $i \geq 0$,

$$
E\left[\sup _{0 \leq s \leq T}\left|\hat{X}_{s}^{0, x_{0} ; \bar{v}, i}\right|^{2}+\sup _{t \leq s \leq T}\left(\left|\hat{X}_{s}^{t, x ; v, i}\right|^{2}+\left|\hat{Y}_{s}^{t, x ; v, i}\right|^{2}\right)+\int_{t}^{T}\left|\hat{Z}_{s}^{t, x ; v, i}\right|^{2} \mathrm{~d} s\right] \leq \alpha C_{0}\left\|\hat{W}^{i}\right\|_{\infty}^{2} .
$$

From (3.8) and (3.9) we see that

$$
E\left[\sup _{0 \leq s \leq T}\left|\hat{X}_{s}^{0, x_{0} ; \bar{v}, i}\right|^{2}+\sup _{t \leq s \leq T}\left(\left|\hat{X}_{s}^{t, x ; v, i}\right|^{2}+\left|\hat{Y}_{s}^{t, x ; v, i}\right|^{2}\right)+\int_{t}^{T}\left|\hat{Z}_{s}^{t, x ; v, i}\right|^{2} \mathrm{~d} s\right] \leq\left(\alpha C_{0}\right)^{i}\left\|\hat{W}^{0}\right\|_{\infty}^{2} .
$$

Notice that $\alpha$ is small enough such that $\alpha C_{0}<1$, thus there exists $\left(X^{0, x_{0} ; \bar{v}}, X^{t, x ; v}, Y^{t, x ; v}, Z^{t, x ; v}\right) \in \mathcal{S}_{\mathbb{F}}^{2}\left(0, T ; \mathbb{R}^{n}\right) \times$ $\mathcal{S}_{\mathbb{F}}^{2}\left(t, T ; \mathbb{R}^{n}\right) \times \mathcal{S}_{\mathbb{F}}^{2}(t, T ; \mathbb{R}) \times \mathcal{H}_{\mathbb{F}}^{2}\left(t, T ; \mathbb{R}^{d}\right)$ such that, as $i \rightarrow \infty$,

$E\left[\sup _{0 \leq s \leq T}\left|X_{s}^{0, x_{0} ; \bar{v}, i}-X_{s}^{0, x_{0} ; \bar{v}}\right|^{2}+\sup _{t \leq s \leq T}\left(\left|X_{s}^{t, x ; v, i}-X_{s}^{t, x ; v}\right|^{2}+\left|Y_{s}^{t, x ; v, i}-Y_{s}^{t, x ; v}\right|^{2}\right)+\int_{t}^{T}\left|Z_{s}^{t, x ; v, i}-Z_{s}^{t, x ; v}\right|^{2} \mathrm{~d} s\right] \rightarrow$ 0 , uniformly in $\bar{v} \in \mathcal{V}_{0, T}, v \in \mathcal{V}_{t, T}$.

Taking the limit in the fully coupled forward-backward SDE (3.7) we get

$$
\left\{\begin{aligned}
\mathrm{d} X_{s}^{t, x ; v}= & E^{\prime}\left[b\left(s,\left(X_{s}^{0, x_{0} ; \bar{v}}\right)^{\prime}, W\left(s,\left(X_{s}^{0, x_{0} ; \bar{v}}\right)^{\prime}\right), X_{s}^{t, x ; v}, Y_{s}^{t, x ; v}, Z_{s}^{t, x ; v}, v_{s}\right)\right] \mathrm{d} s \\
& +E^{\prime}\left[\sigma\left(s,\left(X_{s}^{0, x_{0} ; \bar{v}}\right)^{\prime}, W\left(s,\left(X_{s}^{0, x_{0} ; \bar{v}}\right)^{\prime}\right), X_{s}^{t, x ; v}, Y_{s}^{t, x ; v}, v_{s}\right)\right] \mathrm{d} B_{s}, \\
\mathrm{~d} Y_{s}^{t, x ; v}= & -E^{\prime}\left[f\left(s,\left(X_{s}^{0, x_{0} ; \bar{v}}\right)^{\prime}, W\left(s,\left(X_{s}^{0, x_{0} ; \bar{v}}\right)^{\prime}\right), X_{s}^{t, x ; v}, Y_{s}^{t, x ; v}, Z_{s}^{t, x ; v}, v_{s}\right)\right] \mathrm{d} s \\
& +Z_{s}^{t, x ; v} \mathrm{~d} B_{s}, \\
X_{t}^{t, x ; v}= & x, \quad Y_{T}^{t, x ; v}=E^{\prime}\left[\Phi\left(\left(X_{T}^{0, x_{0} ; \bar{v}}\right)^{\prime}, X_{T}^{t, x ; v}\right)\right] .
\end{aligned}\right.
$$

Finally, by Lemma 3.2 we know that the solution of the system (3.11) is unique.

Step 2. We prove $W=\bar{W} \triangleq \underset{v \in \mathcal{V}_{t, T}}{\operatorname{essinf}} Y_{t}^{t, x ; v}$.

Recall that $W^{i+1}(t, x)=\operatorname{essinf}_{v \in \mathcal{V}_{t, T}} Y_{t}^{t, x ; v, i}=\inf _{v \in \mathcal{V}_{t, T}} E\left[Y_{t}^{t, x ; v, i}\right], i \geq 0$. We conclude by observing that, for $(t, x) \in[0, T] \times \mathbb{R}^{n}$,

$$
\begin{aligned}
\left|W(t, x)-\inf _{v \in \mathcal{V}_{t, T}} E\left[Y_{t}^{t, x ; v}\right]\right| & \leq \sup _{v \in \mathcal{V}_{t, T}} E\left[\left|Y_{t}^{t, x ; v}-Y_{t}^{t, x ; v, i}\right|\right]+\left|W^{i+1}(t, x)-W(t, x)\right| \\
& \leq \sup _{v \in \mathcal{V}_{t, T}} E\left[\left|Y_{t}^{t, x ; v}-Y_{t}^{t, x ; v, i}\right|\right]+\left\|W^{i+1}-W\right\|_{\infty} \rightarrow 0, \text { as } i \rightarrow \infty .
\end{aligned}
$$

Remark 3.8. Indeed, from the proof of Lemma 3.2 and that of Theorem 3.7, we know that $\alpha$ in (H3.1) small enough to satisfy the following conditions:

$$
\left\{\begin{aligned}
1 & >\alpha C_{0}, \\
\beta_{1} & >\sqrt{3}(\tilde{L}+\alpha K)\left(1+2 \mathrm{e}^{\left(4 L^{2}+2(\tilde{L}+\alpha K)^{2}+2 L+1\right) T}\right), \\
\mu_{1} & >\tilde{L}_{\Phi}+4 \sqrt{3}(\tilde{L}+\alpha K)\left(\tilde{L}_{\Phi}^{2}+L_{\Phi}^{2}\right) \mathrm{e}^{\left(4 L^{2}+2(\tilde{L}+\alpha K)^{2}+2 L+1\right) T},
\end{aligned}\right.
$$

where $C_{0}$ equals to $C_{14}$ in Lemma A.3, $\tilde{L}, L, \tilde{L}_{\Phi}, L_{\Phi}$ are the Lipschitz constants of $h=b, \sigma, f$ and $\Phi$ with respect to $x^{\prime}$ and $x$, respectively, and $K$ is the Lipschitz constant of $\tilde{W}$. 


\section{The DYNAmic PRogramming PRINCIPLE}

Peng [16] was the first to introduce the notion of "backward semigroup". From then on, it has been widely used to solve stochastic control problems. Here we extend Peng's concept of "backward semigroups" to controlled fully coupled FBSDEs involving their value function. Let $x_{0} \in \mathbb{R}^{n}, \bar{v} \in \mathcal{V}_{0, T}$ be chosen arbitrarily but fixed. For $(t, x, v) \in[0, T] \times \mathbb{R}^{n} \times \mathcal{V}_{t, T}$ and $0<\delta \leq T-t$, we consider the following fully coupled FBSDE with time horizon $t+\delta$,

$$
\left\{\begin{aligned}
\mathrm{d} \tilde{X}_{s}^{t, x ; v}= & E^{\prime}\left[b\left(s,\left(X_{s}^{0, x_{0} ; \bar{v}}\right)^{\prime}, W\left(s,\left(X_{s}^{0, x_{0} ; \bar{v}}\right)^{\prime}\right), \tilde{X}_{s}^{t, x ; v}, \tilde{Y}_{s}^{t, x ; v}, \tilde{Z}_{s}^{t, x ; v}, v_{s}\right)\right] \mathrm{d} s \\
& +E^{\prime}\left[\sigma\left(s,\left(X_{s}^{0, x_{0} ; \bar{v}}\right)^{\prime}, W\left(s,\left(X_{s}^{0, x_{0} ; \bar{v}}\right)^{\prime}\right), \tilde{X}_{s}^{t, x ; v}, \tilde{Y}_{s}^{t, x ; v}, v_{s}\right)\right] \mathrm{d} B_{s}, \\
\mathrm{~d} \tilde{Y}_{s}^{t, x ; v}= & -E^{\prime}\left[f\left(s,\left(X_{s}^{0, x_{0} ; \bar{v}}\right)^{\prime}, W\left(s,\left(X_{s}^{0, x_{0} ; \bar{v}}\right)^{\prime}\right), \tilde{X}_{s}^{t, x ; v}, \tilde{Y}_{s}^{t, x ; v}, \tilde{Z}_{s}^{t, x ; v}, v_{s}\right)\right] \mathrm{d} s \\
& +\tilde{Z}_{s}^{t, x ; v} \mathrm{~d} B_{s}, \\
\tilde{X}_{t}^{t, x ; v}= & x, \quad \tilde{Y}_{t+\delta}^{t, x ; v}=E^{\prime}\left[\Psi\left(\left(X_{t+\delta}^{0, x_{0} ; \bar{v}}\right)^{\prime}, \tilde{X}_{t+\delta}^{t, x ; v}\right)\right],
\end{aligned}\right.
$$

where $\Psi: \mathbb{R}^{n} \times \mathbb{R}^{n} \rightarrow \mathbb{R}$ is a Lipschitz function with $<G^{T}\left(\Psi\left(x^{\prime}, x\right)-\Psi\left(x^{\prime}, \bar{x}\right)\right), x-\bar{x}>\geq 0$, and $W$ is defined as in Theorem 3.7, and $\left(X^{0, x_{0} ; \bar{v}}, Y^{0, x_{0} ; \bar{v}}, Z^{0, x_{0} ; \bar{v}}\right)$ is the solution of the fully coupled FBSDE (3.1) involving the value function, for our given $x_{0} \in \mathbb{R}^{n}$ and $\bar{v} \in \mathcal{V}_{0, T}$ with time horizon $T$.

Lemma 4.1. Under the assumptions (H3.1), ( $\left.\mathrm{H} 3.2 \mathrm{~L}_{0}\right)$ and $(\mathrm{H} 3.3)$, there is a positive constant $\delta_{0}$ depending on the Lipschitz constant $L$ such that, for $0 \leq \delta \leq \delta_{0}$, the fully coupled FBSDE (4.1) admits a unique solution $\left(\tilde{X}_{s}^{t, x ; v}, \tilde{Y}_{s}^{t, x ; v}, \tilde{Z}_{s}^{t, x ; v}\right)_{s \in[t, t+\delta]} \in \mathcal{S}_{\mathbb{F}}^{2}\left(t, t+\delta ; \mathbb{R}^{n}\right) \times \mathcal{S}_{\mathbb{F}}^{2}(t, t+\delta ; \mathbb{R}) \times \mathcal{H}_{\mathbb{F}}^{2}\left(t, t+\delta ; \mathbb{R}^{d}\right)$.

Proof. From Theorem 3.7 we have the existence of $W$ and $\left(X_{s}^{0, x_{0} ; \bar{v}}\right)_{s \in[0, T]} \in \mathcal{S}_{\mathbb{F}}^{2}\left(0, T ; \mathbb{R}^{n}\right)$. Hence, we can define the (deterministic) functions $h^{W}(s, x, y, z, v)=E\left[h\left(s, X_{s}^{0, x_{0} ; \bar{v}}, W\left(s, X_{s}^{0, x_{0} ; \bar{v}}\right), x, y, z, v\right)\right], h=b, \sigma, f$, respectively, $\Psi^{W}(x)=E\left[\Psi\left(X_{t+\delta}^{0, x_{0} ; \bar{v}}, x\right)\right]$.

Obviously, $h^{W}$ is Lipschitz in $(x, y, z)$, uniformly with respect to $(s, v)$, with the same Lipschitz constant as that of the functions $b, \sigma, f$; and $\Psi^{W}$ is Lipschitz in $x$. According to standard estimates for classical fully coupled FBSDEs (or see Prop. 6.4 in [13] for the general case where $\sigma$ depends on $z$ ), we know that there is a $\delta_{0}>0$ such that, for $s \in[t, t+\delta]$ with $0 \leq \delta \leq \delta_{0}$, the fully coupled FBSDE (4.1) has a unique adapted solution on time horizon $[t, t+\delta]$, denoted by $\left(\tilde{X}_{s}^{t, x ; v}, \tilde{Y}_{s}^{t, x ; v}, \tilde{Z}_{s}^{t, x ; v}\right)_{s \in[t, t+\delta]} \in \mathcal{S}_{\mathbb{F}}^{2}\left(t, t+\delta ; \mathbb{R}^{n}\right) \times \mathcal{S}_{\mathbb{F}}^{2}(t, t+\delta ; \mathbb{R}) \times \mathcal{H}_{\mathbb{F}}^{2}\left(t, t+\delta ; \mathbb{R}^{d}\right)$.

Now we can define the backward semigroup:

$$
G_{s, t+\delta}^{t, x ; v}\left(E^{\prime}\left[\Psi\left(\left(X_{t+\delta}^{0, x_{0} ; \bar{v}}\right)^{\prime}, \tilde{X}_{t+\delta}^{t, x ; v}\right)\right]\right) \triangleq \tilde{Y}_{s}^{t, x ; v}, s \in[t, t+\delta]
$$

where $\left(\tilde{X}_{s}^{t, x ; v}, \tilde{Y}_{s}^{t, x ; v}, \tilde{Z}_{s}^{t, x ; v}\right)_{s \in[t, t+\delta]}$ is the solution of FBSDE (4.1).

Inspired by the work of Li, Wei [13], with the help of the notion of extended "backward semigroup", we can establish the DPP for the value function $W$ defined through the fully coupled FBSDE (3.1).

Theorem 4.2. (DPP) Suppose the assumptions (H3.1), (H3.2 $\left.\mathrm{L}_{0}\right)$ and $(\mathrm{H} 3.3)$ hold. Then there is a positive $\delta_{0}$ depending on $L$ such that, for all $0 \leq t \leq T-\delta$ with $0<\delta \leq \delta_{0}$,

$$
W(t, x)=\underset{v \in \mathcal{V}_{t, t+\delta}}{\operatorname{essinf}} G_{t, t+\delta}^{t, x ; v}\left(W\left(t+\delta, \tilde{X}_{t+\delta}^{t, x ; v}\right)\right) .
$$

The proof of Theorem 4.2 uses the same argument as that of Buckdahn and $\mathrm{Li}[4]$ and is omitted here.

Remark 4.3. According to the definition of backward semigroup, we get, for $t \leq s \leq t+\delta, v \in \mathcal{V}_{t, T}, x \in \mathbb{R}^{n}$,

$$
G_{s, t+\delta}^{t, x ; v}\left(W\left(t+\delta, \tilde{X}_{t+\delta}^{t, x ; v}\right)\right)=\tilde{Y}_{s}^{t, x ; v},
$$

where $\left(\tilde{X}_{s}^{t, x ; v}, \tilde{Y}_{s}^{t, x ; v}, \tilde{Z}_{s}^{t, x ; v}\right)_{s \in[t, t+\delta]}$ is the solution of (4.1) with $\Psi\left(x^{\prime}, x\right)=W(t+\delta, x),\left(x^{\prime}, x\right) \in \mathbb{R}^{n} \times \mathbb{R}^{n}$. 
Proposition 4.4. The value function $W$ introduced in Theorem 3.7 has for some suitable constant $\bar{L}$ the following property of Hölder continuity in $t$ :

$$
|W(t, x)-W(\bar{t}, x)| \leq \bar{L}(1+|x|)|t-\bar{t}|^{\frac{1}{2}}, t, \bar{t} \in[0, T], x \in \mathbb{R}^{n} .
$$

Proof. The functions $b^{W}, \sigma^{W}, f^{W}, \Psi^{W}$ defined in the proof of Lemma 4.1 are jointly continuous and Lipschitz with respect to $(x, y, z)$ and $x$, respectively, with the same Lipschitz constants as those of $b, \sigma, f$ and $\Psi$. Moreover, from (H3.3) we know that for $s \in[0, T], v \in V,\left|h^{W}(s, 0,0,0, v)\right| \leq L_{b}$. Hence, from standard conclusions for classical fully coupled FBSDEs (see Thm. 3.2 in [13]), we have the above estimate.

\section{Viscosity solution of the ASSOCIATED NONLOCAL HJB EQUATion}

In this section, we discuss the associated nonlocal HJB equation and give its probability interpretation in term of the solution of FBSDE (3.1).

Let us consider the PDE

$$
\left\{\begin{array}{l}
\partial_{t} W(t, x)+H\left(t, x, W(t, \cdot), W(t, x), D W(t, x), D^{2} W(t, x)\right)=0,(t, x) \in[0, T) \times \mathbb{R}^{n} \\
W(T, x)=E\left[\Phi\left(X_{T}^{0, x_{0} ; \bar{v}}, x\right)\right], x \in \mathbb{R}^{n}
\end{array}\right.
$$

where the Hamiltonian $H$ is defined by

$$
\begin{aligned}
& H(t, x, \psi(t, \cdot), y, p, X) \\
& \quad=\inf _{v \in V}\left\{\frac{1}{2} \operatorname{tr}\left(\bar{\sigma} \bar{\sigma}^{T}(t, x, \psi(t, \cdot), y, v) X\right)+p \cdot \bar{b}(t, x, \psi(t, \cdot), y, p \cdot \bar{\sigma}, v)+\bar{f}(t, x, \psi(t, \cdot), y, p \cdot \bar{\sigma}, v)\right\},
\end{aligned}
$$

for $t \in[0, T], y \in \mathbb{R}, x, p \in \mathbb{R}^{n}, X \in \mathbf{S}^{d}$ (by $\mathbf{S}^{d}$ we denote all the $d \times d$ symmetric matrix), and $\psi(\cdot)$ is a deterministic Lipschitz function, and

$$
\begin{aligned}
\bar{b}(t, x, \psi(t, \cdot), y, z, v) & =E\left[b\left(t, X_{t}^{0, x_{0} ; \bar{v}}, \psi\left(t, X_{t}^{0, x_{0} ; \bar{v}}\right), x, y, z, v\right)\right] \\
\bar{\sigma}(t, x, \psi(t, \cdot), y, v) & =E\left[\sigma\left(t, X_{t}^{0, x_{0} ; \bar{v}}, \psi\left(t, X_{t}^{0, x_{0} ; \bar{v}}\right), x, y, v\right)\right] \\
\bar{f}(t, x, \psi(t, \cdot), y, z, v) & =E\left[f\left(t, X_{t}^{0, x_{0} ; \bar{v}}, \psi\left(t, X_{t}^{0, x_{0} ; \bar{v}}\right), x, y, z, v\right)\right] .
\end{aligned}
$$

Here $\left(X^{0, x_{0} ; \bar{v}}, Y^{0, x_{0} ; \bar{v}}, Z^{0, x_{0} ; \bar{v}}\right)$ is the solution of the fully coupled FBSDE (3.1) involving the value function for initial time $t=0$, initial value $x=x_{0}$ and fixed $\bar{v} \in \mathcal{V}_{0, T}$.

Let us recall the definition of a viscosity solution of the associated HJB equation (5.1) (see [8] for details).

Definition 5.1. A real-valued continuous function $V \in C\left([0, T] \times \mathbb{R}^{n}\right)$ is called

(i) a viscosity subsolution of equation (5.1), if $V(T, x) \leq E\left[\Phi\left(X_{T}^{0, x_{0} ; \bar{v}}, x\right)\right]$, for all $x \in \mathbb{R}^{n}$, and if for all function $\phi \in C^{1,2}\left([0, T] \times \mathbb{R}^{n}\right)$ and $(t, x) \in[0, T) \times \mathbb{R}^{n}$ such that $V-\phi$ attains a local maximum at $(t, x)$,

$$
\partial_{t} \phi(t, x)+H\left(t, x, V(t, \cdot), V(t, x), D \phi(t, x), D^{2} \phi(t, x)\right) \geq 0
$$

(ii) a viscosity supersolution of equation (5.1), if $V(T, x) \geq E\left[\Phi\left(X_{T}^{0, x_{0} ; \bar{v}}, x\right)\right]$, for all $x \in \mathbb{R}^{n}$, and if for all function $\phi \in C^{1,2}\left([0, T] \times \mathbb{R}^{n}\right)$ and $(t, x) \in[0, T) \times \mathbb{R}^{n}$ such that $V-\phi$ attains a local minimum at $(t, x)$,

$$
\partial_{t} \phi(t, x)+H\left(t, x, V(t, \cdot), V(t, x), D \phi(t, x), D^{2} \phi(t, x)\right) \leq 0
$$

(iii) a viscosity solution of equation (5.1) if it is both a viscosity subsolution and supersolution of equation (5.1).

Now we state the main result of this section. 
Theorem 5.2. Assume that the assumptions $(\mathrm{H} 3.1),\left(\mathrm{H} 3.2 \mathrm{~L}_{0}\right)$ and $(\mathrm{H} 3.3)$ hold true. Then the value function $W(t, x) \in C\left([0, T] \times \mathbb{R}^{n}\right)$ given in Theorem 3.7 is a viscosity solution of HJB equation (5.1).

Proof. According to Theorem 3.7 and Proposition 4.4, we know that the deterministic function $W$ is Lipschitz in $x$ and $\frac{1}{2}$-Hölder continuous in $t$. More precisely, there exists a positive constant $\tilde{L}$ such that, for $x, \bar{x} \in \mathbb{R}^{n}$, $t, \bar{t} \in[0, T]$,

$$
\text { (i) }|W(t, x)-W(t, \bar{x})| \leq \tilde{L}|x-\bar{x}| \text {; (ii) }|W(t, x)-W(\bar{t}, x)| \leq \tilde{L}(1+|x|)|t-\bar{t}|^{\frac{1}{2}}
$$

Consequently, $W(t, x) \in C\left([0, T] \times \mathbb{R}^{n}\right)$ is of linear growth.

The coefficients $\bar{b}(t, x, W(t, \cdot), y, z, v), \bar{\sigma}(t, x, W(t, \cdot), y, v), \bar{f}(t, x, W(t, \cdot), y, z, v)$ and $\bar{\Phi}(x)=E\left[\Phi\left(X_{T}^{0, x_{0} ; \bar{v}}, x\right)\right]$ are Lipschitz in $(x, y, z)$, uniformly in $(t, v)$, and $x$, respectively, and satisfy the monotonicity condition of classical fully coupled FBSDEs. Hence, our fully coupled FBSDE (3.1) involving its value function $W$ can be written as

$$
\left\{\begin{aligned}
\mathrm{d} X_{s}^{t, x ; v} & =\bar{b}\left(s, X_{s}^{t, x ; v}, W(s, \cdot), Y_{s}^{t, x ; v}, Z_{s}^{t, x ; v}, v_{s}\right) \mathrm{d} s+\bar{\sigma}\left(s, X_{s}^{t, x ; v}, W(s, \cdot), Y_{s}^{t, x ; v}, v_{s}\right) \mathrm{d} B_{s}, \\
\mathrm{~d} Y_{s}^{t, x ; v} & =-\bar{f}\left(s, X_{s}^{t, x ; v}, W(s, \cdot), Y_{s}^{t, x ; v}, Z_{s}^{t, x ; v}, v_{s}\right) \mathrm{d} s+Z_{s}^{t, x ; v} \mathrm{~d} B_{s}, \\
X_{t}^{t, x ; v} & =x, \quad Y_{T}^{t, x ; v}=\bar{\Phi}\left(X_{T}^{t, x ; v}\right), \\
W(t, x) & =\operatorname{essinf}_{v \in \mathcal{V}_{t, T}}^{t, x ; v}
\end{aligned}\right.
$$

From the probability interpretation for HJB equations associated with controlled fully coupled FBSDEs (see Thm. $4.1 \mathrm{in} \mathrm{Li}$ and Wei [13]), we know that $W$ is a viscosity solution of HJB equation (5.1).

Theorem 5.3. When $\sigma$ also does not depend on y, under the assumptions $(\mathrm{H} 3.1),\left(\mathrm{H} 3.2 L_{0}\right)$, (H3.3) the value function $W(t, x)$ defined in Theorem 3.7 is the unique viscosity solution of HJB equation (5.1) in the class $\Theta=\left\{\varphi \in C\left([0, T] \times \mathbb{R}^{n}\right): \exists \tilde{A}>0\right.$ such that $\lim |\varphi(t, x)| \exp \left\{-\tilde{A}\left[\log \left(\left(|x|^{2}+1\right)^{\frac{1}{2}}\right)\right]^{2}\right\}=0$, uniformly in $t \in[0, T]\}$.

Proof. Let $W^{i} \in \Theta, i=1,2$, be two viscosity solutions of HJB equation (5.1). We define

$$
\begin{aligned}
\tilde{b}\left(s, x, W^{i}(s, \cdot), y, z, v\right) & =E\left[b\left(s, X_{s}^{0, x_{0} ; \bar{v}}, x, W^{i}\left(s, X_{s}^{0, x_{0} ; \bar{v}}\right), y, z, v\right)\right], \\
\tilde{\sigma}\left(s, x, W^{i}(s, \cdot), v\right) & =E\left[\sigma\left(s, X_{s}^{0, x_{0} ; \bar{v}}, x, W^{i}\left(s, X_{s}^{0, x_{0} ; \bar{v}}\right), v\right)\right], \\
\tilde{f}\left(s, x, W^{i}(s, \cdot), y, z, v\right) & =E\left[f\left(s, X_{s}^{0, x_{0} ; \bar{v}}, x, W^{i}\left(s, X_{s}^{0, x_{0} ; \bar{v}}\right), y, z, v\right)\right], \\
\tilde{\Phi}(x) & =E\left[\Phi\left(X_{T}^{0, x_{0} ; \bar{v}}, x\right)\right],
\end{aligned}
$$

$(s, x, y, z, v) \in[0, T] \times \mathbb{R}^{n} \times \mathbb{R} \times \mathbb{R}^{d} \times V$. Hence $W^{i}(t, x) \in \Theta$ is a viscosity solution of the following HJB equation

$$
\left\{\begin{array}{l}
\partial_{t} W^{i}(t, x)+\inf _{v \in V}\left\{\frac{1}{2} \operatorname{tr}\left(\tilde{\sigma} \tilde{\sigma}^{T}\left(t, x, W^{i}(t, \cdot), v\right) D^{2} W^{i}(t, x)\right)\right. \\
\quad+D W^{i}(t, x) \cdot \tilde{b}\left(t, x, W^{i}(t, \cdot), W^{i}(t, x), D W^{i}(t, x) \cdot \tilde{\sigma}\left(t, x, W^{i}(t, \cdot), v\right), v\right) \\
\left.\quad+\tilde{f}\left(t, x, W^{i}(t, \cdot), W^{i}(t, x), D W^{i}(t, x) \cdot \tilde{\sigma}\left(t, x, W^{i}(t, \cdot), v\right), v\right)\right\}=0,(t, x) \in[0, T) \times \mathbb{R}^{n}, \\
W^{i}(T, x)=\tilde{\Phi}(x), x \in \mathbb{R}^{n} .
\end{array}\right.
$$

Since $\tilde{b}, \tilde{\sigma}, \tilde{f}$ and $\tilde{\Phi}$ satisfy all the assumptions for classical FBSDEs and $W^{i}(t, x) \in \Theta$, i.e., $W^{i}(t, x)$ is of at most linear growth, $W^{i}(t, x)$ possesses the stochastic interpretation as the value function

$$
W^{i}(t, x)=\underset{v \in \mathcal{V}_{t, T}}{\operatorname{essinf}} Y_{t}^{i, t, x ; v},(t, x) \in[0, T] \times \mathbb{R}^{n},
$$


of the optimal control problem for system driven by

$$
\left\{\begin{aligned}
\mathrm{d} X_{s}^{i, t, x ; v} & =\tilde{b}\left(s, X_{s}^{i, t, x ; v}, W^{i}(s, \cdot), Y_{s}^{i, t, x ; v}, Z_{s}^{i, t, x ; v}, v_{s}\right) \mathrm{d} s+\tilde{\sigma}\left(s, X_{s}^{i, t, x ; v}, W^{i}(s, \cdot), v_{s}\right) \mathrm{d} B_{s}, \\
\mathrm{~d} Y_{s}^{i, t, x ; v} & =-\tilde{f}\left(s, X_{s}^{i, t, x ; v}, W^{i}(s, \cdot), Y_{s}^{i, t, x ; v}, Z_{s}^{i, t, x ; v}, v_{s}\right) \mathrm{d} s+Z_{s}^{i, t, x ; v} \mathrm{~d} B_{s}, s \in[t, T], \\
X_{t}^{i, t, x ; v} & =x, \quad Y_{T}^{i, t, x ; v}=\tilde{\Phi}\left(X_{T}^{i, t, x ; v}\right),(t, x) \in[0, T] \times \mathbb{R}^{n}, v \in \mathcal{V}_{t, T}
\end{aligned}\right.
$$

(see, e.g., Thms. 4.1 and 5.1 in Li and Wei [14]). Recall the definitions of $\tilde{b}, \tilde{\sigma}, \tilde{f}$ and $\tilde{\Phi}$, and $W^{i}(t, x)$ is Lipschitz and of linear growth in $x,(5.4)$ is in fact the following equation:

$$
\left\{\begin{aligned}
\mathrm{d} X_{s}^{i, t, x ; v}= & E^{\prime}\left[b\left(s,\left(X_{s}^{i, 0, x_{0} ; \bar{v}}\right)^{\prime}, X_{s}^{i, t, x ; v}, W^{i}\left(s,\left(X_{s}^{i, 0, x_{0} ; \bar{v}}\right)^{\prime}\right), Y_{s}^{i, t, x ; v}, Z_{s}^{i, t, x ; v}, v_{s}\right)\right] \mathrm{d} s \\
& +E^{\prime}\left[\sigma\left(s,\left(X_{s}^{i, 0, x_{0} ; \bar{v}}\right)^{\prime}, X_{s}^{i, t, x ; v}, W^{i}\left(s,\left(X_{s}^{i, 0, x_{0} ; \bar{v}}\right)^{\prime}\right), v_{s}\right)\right] \mathrm{d} B_{s}, \\
\mathrm{~d} Y_{s}^{i, t, x ; v}= & -E^{\prime}\left[f\left(s,\left(X_{s}^{i, 0, x_{0} ; \bar{v}}\right)^{\prime}, X_{s}^{i, t, x ; v}, W^{i}\left(s,\left(X_{s}^{i, 0, x_{0} ; \bar{v}}\right)^{\prime}\right), Y_{s}^{i, t, x ; v}, Z_{s}^{i, t, x ; v}, v_{s}\right)\right] \mathrm{d} s \\
& +Z_{s}^{i, t, x ; v} \mathrm{~d} B_{s}, s \in[t, T], \\
X_{t}^{i, t, x ; v}= & x, \quad Y_{T}^{i, t, x ; v}=E^{\prime}\left[\Phi\left(\left(X_{T}^{i, 0, x_{0} ; \bar{v}}\right)^{\prime}, X_{T}^{i, t, x ; v}\right)\right],(t, x) \in[0, T] \times \mathbb{R}^{n}, v \in \mathcal{V}_{t, T}, \\
W^{i}(t, x)= & \operatorname{essinf}_{v \in \mathcal{V}_{t, T}} Y_{t}^{i, t, x ; v},(t, x) \in[0, T] \times \mathbb{R}^{n} .
\end{aligned}\right.
$$

However, from Theorem 3.7 we know that (5.5) has a unique solution. It means $W^{1}(t, x)=W^{2}(t, x),(t, x) \in$ $[0, T] \times \mathbb{R}^{n}$. The proof is complete.

Example 5.4. Let $n=d=1, G=1$ and $V=\left[0, \frac{\pi}{2}\right]$. Frozen $x_{0} \in \mathbb{R}$ and $\bar{v} \in \mathcal{V}_{0, T}$, for $(t, x) \in[0, T] \times \mathbb{R}$ and $v \in \mathcal{V}_{t, T}$, we first consider the controlled fully coupled mean-field FBSDE:

$$
\left\{\begin{aligned}
\mathrm{d} \bar{X}_{s}^{t, x ; v} & =\left(-\beta \bar{Y}_{s}^{t, x ; v}+v_{s}\right) \mathrm{d} s-\left(\beta \bar{X}_{s}^{t, x ; v}+v_{s}\right) \mathrm{d} B_{s} \\
-\mathrm{d} \bar{Y}_{s}^{t, x ; v} & =\left(\alpha E\left[\arctan \bar{X}_{s}^{0, x_{0} ; \bar{v}}\right]+\beta \bar{X}_{s}^{t, x ; v}-\beta \bar{Z}_{s}^{t, x ; v}-v_{s}\right) \mathrm{d} s-\bar{Z}_{s}^{t, x ; v} \mathrm{~d} B_{s}, \\
\bar{X}_{t}^{t, x ; v} & =x \\
\bar{Y}_{T}^{t, x ; v} & =\bar{X}_{T}^{t, x ; v}
\end{aligned}\right.
$$

where $\beta$ and $\alpha$ satisfy

$$
\left\{\begin{array}{l}
\beta>4 \alpha\left(1+2 \mathrm{e}^{T\left\{4 \beta^{2}+2 \alpha^{2}+2 \beta+2 \alpha+1\right\}}\right), \\
1>16 \alpha \mathrm{e}^{T\left\{4 \beta^{2}+2 \alpha^{2}+2 \beta+2 \alpha+1\right\}} .
\end{array}\right.
$$

We remark that (5.6) does not involve the value function itself. According to Remark 3.5, we know that under the assumption (5.7), the fully coupled FBSDE (5.6) has a unique solution $\left(\bar{X}^{t, x ; v}, \bar{Y}^{t, x ; v}, \bar{Z}^{t, x ; v}\right)$. We define $\bar{W}(t, x) \triangleq \underset{v \in \mathcal{V}_{t, T}}{\operatorname{essinf}} \bar{Y}_{t}^{t, x ; v}$. Applying standard estimate technique for classical fully coupled FBSDEs, we obtain for $t \in[0, T], x, \bar{x} \in \mathbb{R}$,

$$
\text { (i) }|\bar{W}(t, x)-\bar{W}(t, \bar{x})| \leq L_{1}|x-\bar{x}| \text {; (ii) }|\bar{W}(t, x)| \leq L_{2}(1+|x|) \text {, }
$$

where $L_{1}=\left(C_{1} C_{3} \mathrm{e}^{C_{2} C_{3} T}\right)^{\frac{1}{2}}, \quad L_{2}=\left\{\left(C_{4} C_{8}\right) \vee\left(C_{5} C_{8}+C_{7}\right)\right\}^{\frac{1}{2}} \mathrm{e}^{\frac{1}{2} C_{6} C_{8} T}$ and

$$
\begin{aligned}
& C_{1}=3+36 T \beta^{2} \mathrm{e}^{3 T \beta^{2}}, \quad C_{2}=3 T \beta^{2}+36 T^{2} \beta^{4} \mathrm{e}^{3 T \beta^{2}}, \quad C_{3}=\frac{1}{2}(2+T) \mathrm{e}^{\left(4 \beta^{2}+1\right) T}, \\
& C_{4}=3+72 T \beta^{2} \mathrm{e}^{6 T \beta^{2}}, \quad C_{5}=\frac{3}{2} \pi^{2} T^{2}+6 \pi^{2} T+36 \beta^{2} \pi^{2}\left(T^{3}+T^{2}\right) \mathrm{e}^{6 T \beta^{2}}, \quad C_{6}=144 T^{2} \beta^{4} \mathrm{e}^{6 T \beta^{2}}+6 T \beta^{2}, \\
& C_{7}=T \mathrm{e}^{\left(4 \beta^{2}+2 \pi^{2}+1\right) T}, \quad C_{8}=\frac{1}{2}(2+T) \mathrm{e}^{\left(4 \beta^{2}+2 \pi^{2}+1\right) T} .
\end{aligned}
$$


Taking $L_{0}=L_{1} \vee L_{2}$ and choosing $\alpha$ and $\beta$ with

$$
\left\{\begin{array}{l}
\beta>2\left(2+L_{0}\right) \alpha\left(1+2 \mathrm{e}^{T\left\{4 \beta^{2}+2 \alpha^{2}\left(1+L_{0}\right)^{2}+2 \beta+2 \alpha+1\right\}}\right), \\
1>8\left(2+L_{0}\right) \alpha \mathrm{e}^{T\left\{4 \beta^{2}+2 \alpha^{2}\left(1+L_{0}\right)^{2}+2 \beta+2 \alpha+1\right\}},
\end{array}\right.
$$

from Theorem 3.7, the following FBSDE involving the value function:

$$
\left\{\begin{aligned}
\mathrm{d} X_{s}^{t, x ; v} & =\left(-\alpha E\left[\arctan W\left(s, X_{s}^{0, x_{0} ; \bar{v}}\right)\right]-\beta Y_{s}^{t, x ; v}+v_{s}\right) \mathrm{d} s-\left(\beta X_{s}^{t, x ; v}+v_{s}\right) \mathrm{d} B_{s}, \\
-\mathrm{d} Y_{s}^{t, x ; v} & =\left(\alpha E\left[\arctan X_{s}^{0, x_{0} ; \bar{v}}\right]+\beta X_{s}^{t, x ; v}-\beta Z_{s}^{t, x ; v}-v_{s}\right) \mathrm{d} s-Z_{s}^{t, x ; v} \mathrm{~d} B_{s}, \\
X_{t}^{t, x ; v} & =x, \quad Y_{T}^{t, x ; v}=X_{T}^{t, x ; v} \\
W(t, x) & =\underset{v \in \mathcal{V}_{t, T}}{\operatorname{essinf}} Y_{t}^{t, x ; v}
\end{aligned}\right.
$$

has a unique solution, denoted by $\left(X^{t, x ; v}, Y^{t, x ; v}, Z^{t, x ; v}, W\right)$. Moreover, from Theorem 5.2 the value function $W$ is a viscosity solution of the nonlocal HJB equation:

$$
\left\{\begin{array}{l}
\partial_{t} W+\inf _{v \in\left[0, \frac{\pi}{2}\right]}\left\{\frac{1}{2}(\beta x+v)^{2} \partial_{x x} W-\left(\alpha E\left[\arctan W\left(t, X_{t}^{0, x_{0} ; \bar{v}}\right)\right]+\beta W-\beta^{2} x-(\beta+1) v\right) \partial_{x} W\right. \\
\left.\quad+\alpha E\left[\arctan X_{t}^{0, x_{0} ; \bar{v}}\right]+\beta x-v\right\}=0, \quad(t, x) \in[0, T] \times \mathbb{R} \\
W(T, x)=x, \quad x \in \mathbb{R} .
\end{array}\right.
$$

\section{APPENDIX}

In this section we prove some auxiliary lemmas for Theorem 3.7.

Let $W^{i} \in \mathcal{W}$. In order to make notations concisely, we denote by $\left(X^{i}, Y^{i}, Z^{i}\right)$ (resp., $\left.\left(X^{0, i}, Y^{0, i}, Z^{0, i}\right)\right)$ the solution of the fully coupled FBSDE (3.7) for $(t, x, v) \in[0, T] \times \mathbb{R}^{n} \times \mathcal{V}_{t, T}$ (resp., for $(t, x, v)=\left(0, x_{0}, \bar{v}\right)$ ).

For any $(t, x) \in[0, T] \times \mathbb{R}^{n}$ and $v \in \mathcal{V}_{t, T}$, we set, $\hat{X}^{i} \triangleq X^{i+1}-X^{i}, \hat{Y}^{i} \triangleq Y^{i+1}-Y^{i}, \hat{Z}^{i} \triangleq Z^{i+1}-Z^{i}, \hat{W}^{i}(t, x) \triangleq$ $W^{i+1}(t, x)-W^{i}(t, x), \quad i \geq 0$. Accordingly, $\hat{X}^{0, i} \triangleq X^{0, i+1}-X^{0, i}, \quad \hat{Y}^{0, i} \triangleq Y^{0, i+1}-Y^{0, i}, \quad \hat{Z}^{0, i} \triangleq Z^{0, i+1}-Z^{0, i}$.

Lemma A.1. Under the assumptions $(\mathrm{H} 3.1),\left(\mathrm{H} 3.2 \mathrm{~L}_{0}\right)$ and $(\mathrm{H} 3.3)$, for any $\varepsilon>0$, there exists some $M$ large enough such that, for all $i \in \mathbb{N}$,

$$
E \int_{0}^{T}\left|\hat{W}^{i}\left(s, X_{s}^{0, i}\right)\right|^{2} \mathrm{~d} s \leq T|| \hat{W}^{i} \|_{K_{M}}^{2}+\varepsilon
$$

Proof. For arbitrarily given $M>0$, we have, for all $i \geq 0$,

$$
\begin{aligned}
& E \int_{0}^{T}\left|\hat{W}^{i}\left(s, X_{s}^{0, i}\right)\right|^{2} \mathrm{~d} s \leq \int_{0}^{T}\left\|\hat{W}^{i}\right\|_{K_{M}}^{2} \mathrm{~d} s+E \int_{0}^{T}\left|\hat{W}^{i}\left(s, X_{s}^{0, i}\right)\right|^{2} I_{\left\{\left|X_{s}^{0, i}\right|>M\right\}} \mathrm{d} s \\
& \leq T|| \hat{W}^{i}\left\|_{K_{M}}^{2}+8 L_{0}^{2} E \int_{0}^{T}\left(1+\left|X_{s}^{0, i}\right|^{2}\right) I_{\left\{\left|X_{s}^{0, i}\right|>M\right\}} \mathrm{d} s \leq T|| \hat{W}^{i}\right\|_{K_{M}}^{2}+\frac{8 L_{0}^{2}}{M} E \int_{0}^{T}\left(\left|X_{s}^{0, i}\right|+\left|X_{s}^{0, i}\right|^{3}\right) \mathrm{d} s .
\end{aligned}
$$

From Lemma 3.3 (ii), for $(t, x, v)=\left(0, x_{0}, \bar{v}\right), \sup _{i \geq 0} E\left[\int_{0}^{T}\left(\left|X_{s}^{0, i}\right|+\left|X_{s}^{0, i}\right|^{3}\right) \mathrm{d} s\right] \leq T L_{0}^{3}\left(1+\left|x_{0}\right|+\left|x_{0}\right|^{3}\right)$.

Hence, for any $\varepsilon>0$, there exists some $M$ enough large such that, for all $i \in N$,

$$
E \int_{0}^{T}\left|\hat{W}^{i}\left(s, X_{s}^{0, i}\right)\right|^{2} \mathrm{~d} s \leq T|| \hat{W}^{i} \|_{K_{M}}^{2}+\varepsilon
$$


Lemma A.2. We suppose that the assumptions (H3.1), (H3.2 $\left.\mathrm{L}_{0}\right),(\mathrm{H} 3.3)$ hold true. Then there exists a constant $C_{0}>0$ depending only on $\tilde{L}, L, L_{0}, L_{\Phi}, \tilde{L}_{\Phi}, \alpha$ such that

$$
E\left[\sup _{0 \leq s \leq T}\left(\left|\hat{X}_{s}^{0, i}\right|^{2}+\left|\hat{Y}_{s}^{0, i}\right|^{2}\right)+\int_{0}^{T}\left|\hat{Z}_{s}^{0, i}\right|^{2} \mathrm{~d} s\right] \leq \alpha C_{0}\left(\left\|\hat{W}^{i}\right\|_{K_{M}}^{2}+\varepsilon\right) .
$$

Proof. For the forward SDE of $(3.7)$ and $(t, x, v)=\left(0, x_{0}, \bar{v}\right), i \geq 0$, we have from standard estimates and Lemma A.1 that

$$
E\left[\sup _{0 \leq s \leq T}\left|\hat{X}_{s}^{0, i}\right|^{2}\right] \leq C_{0} T \alpha^{2}\left(\left\|\hat{W}^{i}\right\|_{K_{M}}^{2}+\varepsilon\right)+C_{0} L^{2} E \int_{0}^{T}\left(\left|\hat{Y}_{s}^{0, i}\right|^{2}+\left|\hat{Z}_{s}^{0, i}\right|^{2}\right) \mathrm{d} s,
$$

where $C_{0}=(10 T+32)\left(1+\kappa \mathrm{e}^{\kappa}\right)$ and $\kappa=(10 T+8)\left\{\left(\tilde{L}+\alpha L_{0}\right)^{2}+L^{2}\right\} T$.

To estimate the backward SDE of (3.7) for $(t, x, v)=\left(0, x_{0}, \bar{v}\right)$, we apply the Itô's formula to $\mathrm{e}^{\beta s}\left|\hat{Y}_{s}^{0, i}\right|^{2}$ (Recall that $\left.\beta=4 L^{2}+2\left(\tilde{L}+\alpha L_{0}\right)^{2}+2 L+2 \alpha+1\right)$. Then, thanks to Lemma (A.1) and to standard estimates we have, for $0 \leq s \leq T, i \geq 0$,

$$
E\left[\left|\hat{Y}_{s}^{0, i}\right|^{2}+\int_{s}^{T}\left(\left|\hat{Y}_{r}^{0, i}\right|^{2}+\left|\hat{Z}_{r}^{0, i}\right|^{2}\right) \mathrm{d} r\right] \leq 4\left(\tilde{L}_{\Phi}^{2}+L_{\Phi}^{2}\right) \mathrm{e}^{\beta T} E\left|\hat{X}_{T}^{0, i}\right|^{2}+2 \mathrm{e}^{\beta T} E \int_{s}^{T}\left|\hat{X}_{r}^{0, i}\right|^{2} \mathrm{~d} r+\alpha T \mathrm{e}^{\beta T}\left(\| \hat{W}^{i}||_{K_{M}}^{2}+\varepsilon\right) .
$$

On the other hand,

$$
\begin{aligned}
E\left[\left\langle\hat{X}_{T}^{0, i}, G^{T} \hat{Y}_{T}^{0, i}\right\rangle\right]= & E\left[E^{\prime}\left[\left\langle\hat{X}_{T}^{0, i}, G^{T}\left(\Phi\left(\left(X_{T}^{0, i+1}\right)^{\prime}, X_{T}^{0, i+1}\right)-\Phi\left(\left(X_{T}^{0, i}\right)^{\prime}, X_{T}^{0, i}\right)\right)\right\rangle\right]\right] \\
& \geq\left(\mu_{1}-\tilde{L}_{\Phi}\right) E\left|\hat{X}_{T}^{0, i}\right|^{2} .
\end{aligned}
$$

We denote

$$
\hat{B}^{0, i}(s)=\left(\begin{array}{c}
-G^{T} \hat{f}_{s}^{0, i} \\
G \hat{b}_{s}^{0, i} \\
G \hat{\sigma}_{s}^{0, i}
\end{array}\right)
$$

where

$$
\begin{aligned}
\hat{f}_{s}^{0, i}= & E^{\prime}\left[f\left(s,\left(X_{s}^{0, i+1}\right)^{\prime}, W^{i+1}\left(s,\left(X_{s}^{0, i+1}\right)^{\prime}\right), X_{s}^{0, i+1}, Y_{s}^{0, i+1}, Z_{s}^{0, i+1}, \bar{v}_{s}\right)\right] \\
& -E^{\prime}\left[f\left(s,\left(X_{s}^{0, i}\right)^{\prime}, W^{i}\left(s,\left(X_{s}^{0, i}\right)^{\prime}\right), X_{s}^{0, i}, Y_{s}^{0, i}, Z_{s}^{0, i}, \bar{v}_{s}\right)\right], \\
\hat{b}_{s}^{0, i}= & E^{\prime}\left[b\left(s,\left(X_{s}^{0, i+1}\right)^{\prime}, W^{i+1}\left(s,\left(X_{s}^{0, i+1}\right)^{\prime}\right), X_{s}^{0, i+1}, Y_{s}^{0, i+1}, Z_{s}^{0, i+1}, \bar{v}_{s}\right)\right] \\
& -E^{\prime}\left[b\left(s,\left(X_{s}^{0, i}\right)^{\prime}, W^{i}\left(s,\left(X_{s}^{0, i}\right)^{\prime}\right), X_{s}^{0, i}, Y_{s}^{0, i}, Z_{s}^{0, i}, \bar{v}_{s}\right)\right], \\
\hat{\sigma}_{s}^{0, i}= & E^{\prime}\left[\sigma\left(s,\left(X_{s}^{0, i+1}\right)^{\prime}, W^{i+1}\left(s,\left(X_{s}^{0, i+1}\right)^{\prime}\right), X_{s}^{0, i+1}, Y_{s}^{0, i+1}, \bar{v}_{s}\right)\right] \\
& -E^{\prime}\left[\sigma\left(s,\left(X_{s}^{0, i}\right)^{\prime}, W^{i}\left(s,\left(X_{s}^{0, i}\right)^{\prime}\right), X_{s}^{0, i}, Y_{s}^{0, i}, \bar{v}_{s}\right)\right] .
\end{aligned}
$$

Then, due to $(\mathrm{H} 3.1)$ and $\left(\mathrm{H} 3.2 \mathrm{~L}_{0}\right)$,

$$
\begin{aligned}
& \left|E\left[\left\langle\hat{B}^{0, i}(s),\left(\hat{X}_{s}^{0, i}, \hat{Y}_{s}^{0, i}, \hat{Z}_{s}^{0, i}\right)\right\rangle\right]\right| \\
& \leq E\left[-\beta_{1}\left|\hat{X}_{s}^{0, i}\right|^{2}\right]+E\left[\sqrt{3}\left\{\left(\tilde{L}+\alpha L_{0}\right) E^{\prime}\left|\widehat{\left(X_{s}^{0, i}\right)^{\prime} \mid}+\alpha E^{\prime}\right| \hat{W}^{i}\left(s,\left(X_{s}^{0, i}\right)^{\prime}\right) \mid\right\} \sqrt{\left|\hat{X}_{s}^{0, i}\right|^{2}+\left|\hat{Y}_{s}^{0, i}\right|^{2}+\left|\hat{Z}_{s}^{0, i}\right|^{2}}\right] .
\end{aligned}
$$

Hence, we have, for $0 \leq s \leq T$,

$$
\begin{gathered}
E\left[\left\langle\hat{X}_{s}^{0, i}, G^{T} \hat{Y}_{s}^{0, i}\right\rangle\right]=E\left[\left\langle\hat{X}_{T}^{0, i}, G^{T} \hat{Y}_{T}^{0, i}\right\rangle\right]-E\left[\int_{s}^{T}\left\langle\hat{B}^{0, i}(r),\left(\hat{X}_{r}^{0, i}, \hat{Y}_{r}^{0, i}, \hat{Z}_{r}^{0, i}\right)\right\rangle \mathrm{d} r\right] \\
\geq\left(\mu_{1}-\tilde{L}_{\Phi}\right) E\left|\hat{X}_{T}^{0, i}\right|^{2}+\left(\beta_{1}-2\left(\tilde{L}+\alpha L_{0}+\alpha\right)\right) E \int_{s}^{T}\left|\hat{X}_{r}^{0, i}\right|^{2} \mathrm{~d} r \\
-\frac{1}{2}\left(\tilde{L}+\alpha L_{0}+\alpha\right) E \int_{s}^{T}\left(\left|\hat{Y}_{r}^{0, i}\right|^{2}+\left|\hat{Z}_{r}^{0, i}\right|^{2}\right) \mathrm{d} r-\frac{3}{2} \alpha T\left(\left\|\hat{W}^{i}\right\|_{K_{M}}^{2}+\varepsilon\right) .
\end{gathered}
$$


From (A.3) and (A.4),

$$
\begin{aligned}
E & {\left[\left\langle\hat{X}_{s}^{0, i}, G^{T} \hat{Y}_{s}^{0, i}\right\rangle\right] \geq\left(\mu_{1}-\tilde{L}_{\Phi}-2\left(\tilde{L}+\alpha L_{0}+\alpha\right)\left(\tilde{L}_{\Phi}^{2}+L_{\Phi}^{2}\right) \mathrm{e}^{\beta T}\right) E\left|\hat{X}_{T}^{0, i}\right|^{2} } \\
& +\left\{\beta_{1}-2\left(\tilde{L}+\alpha L_{0}+\alpha\right)\left(1+\frac{1}{2} \mathrm{e}^{\beta T}\right)\right\} E \int_{s}^{T}\left|\hat{X}_{r}^{0, i}\right|^{2} \mathrm{~d} r-\alpha\left(\frac{3}{2} T+\frac{1}{2} T\left(\tilde{L}+\alpha L_{0}+\alpha\right) \mathrm{e}^{\beta T}\right)\left(\left\|\hat{W}^{i}\right\|_{K_{M}}^{2}+\varepsilon\right),
\end{aligned}
$$

where $\beta$ is given in $\left(\mathrm{H} 3.2 \mathrm{~L}_{0}\right)$.

Since $\beta_{1}$ and $\mu_{1}$ satisfy

$$
\begin{aligned}
& \left.\left.\beta_{1}\right\rangle 2\left(\tilde{L}+\alpha L_{0}+\alpha\right)\left(1+2 \mathrm{e}^{\beta T}\right)\right\rangle 2\left(\tilde{L}+\alpha L_{0}+\alpha\right)\left(1+\frac{1}{2} \mathrm{e}^{\beta T}\right), \\
& \left.\left.\mu_{1}\right\rangle \tilde{L}_{\Phi}+8\left(\tilde{L}+\alpha L_{0}+\alpha\right)\left(\tilde{L}_{\Phi}^{2}+L_{\Phi}^{2}\right) \mathrm{e}^{\beta T}\right\rangle \tilde{L}_{\Phi}+2\left(\tilde{L}+\alpha L_{0}+\alpha\right)\left(\tilde{L}_{\Phi}^{2}+L_{\Phi}^{2}\right) \mathrm{e}^{\beta T},
\end{aligned}
$$

we have, for $s=0$,

$$
E \int_{0}^{T}\left|\hat{X}_{r}^{0, i}\right|^{2} \mathrm{~d} r \leq \alpha C_{1}\left(\left\|\hat{W}^{i}\right\|_{K_{M}}^{2}+\varepsilon\right)
$$

and

$$
E\left|\hat{X}_{T}^{0, i}\right|^{2} \leq \alpha C_{2}\left(\left\|\hat{W}^{i}\right\|_{K_{M}}^{2}+\varepsilon\right)
$$

where

$$
C_{1}=\frac{\frac{3}{2} T+\frac{1}{2} T\left(\tilde{L}+\alpha L_{0}+\alpha\right) \mathrm{e}^{\beta T}}{\beta_{1}-2\left(\tilde{L}+\alpha L_{0}+\alpha\right)\left(1+\frac{1}{2} \mathrm{e}^{\beta T}\right)}, \quad C_{2}=\frac{\frac{3}{2} T+\frac{1}{2} T\left(\tilde{L}+\alpha L_{0}+\alpha\right) \mathrm{e}^{\beta T}}{\mu_{1}-\tilde{L}_{\Phi}-2\left(\tilde{L}+\alpha L_{0}+\alpha\right)\left(\tilde{L}_{\Phi}^{2}+L_{\Phi}^{2}\right) \mathrm{e}^{\beta T}} .
$$

According to (A.6), (A.7) and (A.3), for $0 \leq s \leq T$,

$$
E\left|\hat{Y}_{s}^{0, i}\right|^{2}+E \int_{s}^{T}\left(\left|\hat{Y}_{r}^{0, i}\right|^{2}+\left|\hat{Z}_{r}^{0, i}\right|^{2}\right) \mathrm{d} r \leq \alpha C_{3}\left(\left\|\hat{W}^{i}\right\|_{K_{M}}^{2}+\varepsilon\right),
$$

where $C_{3}=4\left(\tilde{L}_{\Phi}^{2}+L_{\Phi}^{2}\right) \mathrm{e}^{\beta T} C_{2}+2 \mathrm{e}^{\beta T} C_{1}+T \mathrm{e}^{\beta T}$.

From (A.2) it follows

$$
E\left[\sup _{0 \leq s \leq T}\left|\hat{X}_{s}^{0, i}\right|^{2}\right] \leq \alpha C_{4}\left(\left\|\hat{W}^{i}\right\|_{K_{M}}^{2}+\varepsilon\right),
$$

where $C_{4}=C_{0}\left(T \alpha+C_{3} L^{2}\right)$. Estimating the backward SDE in (3.7), we have

$$
\begin{aligned}
E & {\left[\sup _{0 \leq s \leq T}\left|\hat{Y}_{s}^{0, i}\right|^{2}+\int_{0}^{T}\left|\hat{Z}_{s}^{0, i}\right|^{2} \mathrm{~d} s\right] \leq 6\left(\tilde{L}_{\Phi}^{2}+L_{\Phi}^{2}\right) E\left|\hat{X}_{T}^{0, i}\right|^{2}+15 T\left\{\left(\tilde{L}+\alpha L_{0}\right)^{2}+L^{2}\right\} E\left[\int_{0}^{T}\left|\hat{X}_{s}^{0, i}\right|^{2} \mathrm{~d} s\right] } \\
& +\left(15 T L^{2}+13\right) E\left[\int_{0}^{T}\left(\left|\hat{Y}_{s}^{0, i}\right|^{2}+\left|\hat{Z}_{s}^{0, i}\right|^{2}\right) \mathrm{d} s\right]+15 T^{2} \alpha^{2}\left(\left\|\hat{W}^{i}\right\|_{K_{M}}^{2}+\varepsilon\right) .
\end{aligned}
$$

From (A.6)-(A.10),

$$
E\left[\sup _{0 \leq s \leq T}\left(\left|\hat{X}_{s}^{0, i}\right|^{2}+\left|\hat{Y}_{s}^{0, i}\right|^{2}\right)+\int_{0}^{T}\left|\hat{Z}_{s}^{0, i}\right|^{2} \mathrm{~d} s\right] \leq \alpha C_{5}\left(\left\|\hat{W}^{i}\right\|_{K_{M}}^{2}+\varepsilon\right),
$$

where $C_{5}=6\left(\tilde{L}_{\Phi}^{2}+L_{\Phi}^{2}\right) C_{2}+15 T\left\{\left(\tilde{L}+\alpha L_{0}\right)^{2}+L^{2}\right\} C_{1}+\left(15 T L^{2}+13\right) C_{3}+15 T^{2} \alpha+C_{4}$.

For the difference of solution $\left(X^{i}, Y^{i}, Z^{i}\right)$, we have the similar estimate.

In the following lemma, we still use the notations $C_{1}$ to $C_{5}$ in Lemma A.2. 
Lemma A.3. We make the same assumptions as in Lemma A.2. Then there exists a $C_{0}>0$ depending only on $\tilde{L}, L, L_{0}, L_{\Phi}, \tilde{L}_{\Phi}, \alpha$ such that, for $(t, x) \in[0, T] \times \mathbb{R}^{n}$ and $v \in \mathcal{V}_{t, T}$,

$$
E\left[\sup _{t \leq s \leq T}\left(\left|\hat{X}_{s}^{i}\right|^{2}+\left|\hat{Y}_{s}^{i}\right|^{2}\right)+\int_{t}^{T}\left|\hat{Z}_{s}^{i}\right|^{2} \mathrm{~d} s\right] \leq \alpha C_{0}\left(\left\|\hat{W}^{i}\right\|_{K_{M}}^{2}+\varepsilon\right) .
$$

Proof. From (3.7) and Lemma A.2 we have, for $0 \leq t \leq s \leq T, i \geq 0$,

$$
E\left[\sup _{t \leq s \leq T}\left|\hat{X}_{s}^{i}\right|^{2}\right] \leq \alpha C_{6}\left(\left\|\hat{W}^{i}\right\|_{K_{M}}^{2}+\varepsilon\right)+C_{7} E \int_{t}^{T}\left(\left|\hat{Y}_{r}^{i}\right|^{2}+\left|\hat{Z}_{r}^{i}\right|^{2}\right) \mathrm{d} r
$$

and

$$
E\left|\hat{Y}_{s}^{i}\right|^{2}+E \int_{s}^{T}\left(\left|\hat{Y}_{r}^{i}\right|^{2}+\left|\hat{Z}_{r}^{i}\right|^{2}\right) \mathrm{d} r \leq 4 \mathrm{e}^{\beta T} L_{\Phi}^{2} E\left|\hat{X}_{T}^{i}\right|^{2}+2 \mathrm{e}^{\beta T} E \int_{s}^{T}\left|\hat{X}_{r}^{i}\right|^{2} \mathrm{~d} r+\alpha C_{8}\left(\left\|\hat{W}^{i}\right\|_{K_{M}}^{2}+\varepsilon\right),
$$

where

$$
\begin{aligned}
& C_{6}=(10 T+32) T\left\{\left(\tilde{L}+\alpha L_{0}\right)^{2} C_{4}+\alpha+L^{2} \mathrm{e}^{(10 T+8) L^{2} T}(10 T+8) T\left[\left(\tilde{L}+\alpha L_{0}\right)^{2} C_{4}+\alpha\right]\right\}, \\
& C_{7}=(10 T+32) L^{2}\left[1+(10 T+8) L^{2} T \mathrm{e}^{(10 T+8) L^{2} T}\right], C_{8}=T \mathrm{e}^{\beta T}+4 \mathrm{e}^{\beta T} \tilde{L}_{\Phi}^{2} C_{2}+\mathrm{e}^{\beta T} C_{4} T .
\end{aligned}
$$

Furthermore, from our assumption (H3.1) and $\left(\mathrm{H} 3.2 \mathrm{~L}_{0}\right)$ as well as (A.7) we obtain, for $i \geq 0$,

$$
\begin{gathered}
E\left[\left\langle\hat{X}_{T}^{i}, \hat{Y}_{T}^{i}\right\rangle\right]=E\left[E^{\prime}\left[\left\langle\hat{X}_{T}^{i}, \Phi\left(\left(X_{T}^{0, i+1}\right)^{\prime}, X_{T}^{i+1}\right)-\Phi\left(\left(X_{T}^{0, i}\right)^{\prime}, X_{T}^{i}\right)\right\rangle\right]\right] \\
\geq \mu_{1} E\left[\left|\hat{X}_{T}^{i}\right|^{2}\right]-\tilde{L}_{\Phi} E\left[\left|\hat{X}_{T}^{0, i}\right|\right] E\left[\left|\hat{X}_{T}^{i}\right|\right] \geq\left(\mu_{1}-\tilde{L}_{\Phi}\right) E\left[\left|\hat{X}_{T}^{i}\right|^{2}\right]-\frac{1}{4} \tilde{L}_{\Phi} E\left[\left|\hat{X}_{T}^{0, i}\right|^{2}\right] \\
\geq\left(\mu_{1}-\tilde{L}_{\Phi}\right) E\left[\left|\hat{X}_{T}^{i}\right|^{2}\right]-\frac{1}{4} \tilde{L}_{\Phi} C_{2} \alpha\left(\|\left.\hat{W}^{i}\right|_{K_{M}} ^{2}+\varepsilon\right) .
\end{gathered}
$$

Let us put

$$
\hat{B}^{i}(s) \triangleq\left(\begin{array}{c}
-G^{T} \hat{f}_{s}^{i} \\
G \hat{b}_{s}^{i} \\
G \hat{\sigma}_{s}^{i}
\end{array}\right)
$$

where $\hat{f}^{i}, \hat{b}^{i}, \hat{\sigma}^{i}$ are defined as follows:

$$
\begin{aligned}
\hat{f}_{s}^{i}= & E^{\prime}\left[f\left(s,\left(X_{s}^{0, i+1}\right)^{\prime}, W^{i+1}\left(s,\left(X_{s}^{0, i+1}\right)^{\prime}\right), X_{s}^{i+1}, Y_{s}^{i+1}, Z_{s}^{i+1}, v_{s}\right)\right] \\
& -E^{\prime}\left[f\left(s,\left(X_{s}^{0, i}\right)^{\prime}, W^{i}\left(s,\left(X_{s}^{0, i}\right)^{\prime}\right), X_{s}^{i}, Y_{s}^{i}, Z_{s}^{i}, v_{s}\right)\right], \\
\hat{b}_{s}^{i}= & E^{\prime}\left[b\left(s,\left(X_{s}^{0, i+1}\right)^{\prime}, W^{i+1}\left(s,\left(X_{s}^{0, i+1}\right)^{\prime}\right), X_{s}^{i+1}, Y_{s}^{i+1}, Z_{s}^{i+1}, v_{s}\right)\right] \\
& -E^{\prime}\left[b\left(s,\left(X_{s}^{0, i}\right)^{\prime}, W^{i}\left(s,\left(X_{s}^{0, i}\right)^{\prime}\right), X_{s}^{i}, Y_{s}^{i}, Z_{s}^{i}, v_{s}\right)\right], \\
\hat{\sigma}_{s}^{i}= & E^{\prime}\left[\sigma\left(s,\left(X_{s}^{0, i+1}\right)^{\prime}, W^{i+1}\left(s,\left(X_{s}^{0, i+1}\right)^{\prime}\right), X_{s}^{i+1}, Y_{s}^{i+1}, v_{s}\right)\right] \\
& -E^{\prime}\left[\sigma\left(s,\left(X_{s}^{0, i}\right)^{\prime}, W^{i}\left(s,\left(X_{s}^{0, i}\right)^{\prime}\right), X_{s}^{i}, Y_{s}^{i}, v_{s}\right)\right] .
\end{aligned}
$$

Then, due to assumptions (H3.2 $\left.\mathrm{L}_{0}\right)$ and (H3.1) (Recall that $W^{i} \in \mathcal{W}, i \geq 0$ ),

$$
\begin{aligned}
\left|E\left[\left\langle\hat{B}^{i}(s),\left(\hat{X}_{s}^{i}, \hat{Y}_{s}^{i}, \hat{Z}_{s}^{i}\right)\right\rangle\right]\right| \leq & -\beta_{1} E\left[\left|\hat{X}_{s}^{i}\right|^{2}\right]+E\left[\sqrt { 3 } \left\{\left(\tilde{L}+\alpha L_{0}\right) E^{\prime}\left[\mid \widehat{\left(X_{s}^{0, i}\right)^{\prime} \mid}\right]\right.\right. \\
& \left.\left.+\alpha E^{\prime}\left|\hat{W}^{i}\left(s,\left(X_{s}^{0, i}\right)^{\prime}\right)\right|\right\} \sqrt{\left|X_{s}^{i}\right|^{2}+\left|Y_{s}^{i}\right|^{2}+\left|Z_{s}^{i}\right|^{2}}\right] .
\end{aligned}
$$


Consequently, from (A.14) we have, for $t \leq s \leq T, i \geq 0$,

$$
\begin{aligned}
& E\left[\left\langle\hat{X}_{s}^{i}, \hat{Y}_{s}^{i}\right\rangle\right]=E\left[\left\langle\hat{X}_{T}^{i}, \hat{Y}_{T}^{i}\right\rangle\right]-E\left[\int_{s}^{T}\left\langle\hat{B}^{i}(r),\left(\hat{X}_{r}^{i}, \hat{Y}_{r}^{i}, \hat{Z}_{r}^{i}\right)>\mathrm{d} r\right]\right. \\
& \geq\left(\mu_{1}-\tilde{L}_{\Phi}\right) E\left|\hat{X}_{T}^{i}\right|^{2}+\left(\beta_{1}-2\left(\tilde{L}+\alpha L_{0}+\alpha\right)\right) E \int_{s}^{T}\left|\hat{X}_{r}^{i}\right|^{2} \mathrm{~d} r \\
& \quad-2\left(\tilde{L}+\alpha L_{0}+\alpha\right) E \int_{s}^{T}\left(\left|\hat{Y}_{r}^{i}\right|^{2}+\left|\hat{Z}_{r}^{i}\right|^{2}\right) \mathrm{d} r-\alpha C_{9}\left(|| \hat{W}^{i} \|_{K_{M}}^{2}+\varepsilon\right),
\end{aligned}
$$

where $C_{9}=\frac{1}{4} \tilde{L}_{\Phi} C_{2}+\frac{3}{8}\left(\tilde{L}+\alpha L_{0}\right) C_{1}+\frac{3}{8} T$.

Thanks to (A.13) and (A.15), it follows

$$
\begin{aligned}
E\left[\left\langle\hat{X}_{s}^{i}, \hat{Y}_{s}^{i}\right\rangle\right] \geq & \left(\mu_{1}-\tilde{L}_{\Phi}-8\left(\tilde{L}+\alpha L_{0}+\alpha\right) L_{\Phi}^{2} \mathrm{e}^{\beta T}\right) E\left|\hat{X}_{T}^{i}\right|^{2} \\
& +\left\{\beta_{1}-2\left(\tilde{L}+\alpha L_{0}+\alpha\right)\left(1+2 \mathrm{e}^{\beta T}\right)\right\} E \int_{s}^{T}\left|\hat{X}_{r}^{i}\right|^{2} \mathrm{~d} r-\alpha C_{10}\left(\left\|\hat{W}^{i}\right\|_{K_{M}}^{2}+\varepsilon\right),
\end{aligned}
$$

where $\beta$ and $\beta_{1}, \mu_{1}$ are given in $\left(\mathrm{H} 3.2 \mathrm{~L}_{0}\right)$, respectively, and $C_{10}=2\left(\tilde{L}+\alpha L_{0}+\alpha\right) C_{8}+C_{9}$.

Therefore, as $s=t$, for $i \geq 0$,

$$
\left\{\begin{array}{l}
E\left|\hat{X}_{T}^{i}\right|^{2} \leq \alpha C_{11}\left(\left\|\hat{W}^{i}\right\|_{K_{M}}^{2}+\varepsilon\right), \\
E \int_{t}^{T}\left|\hat{X}_{r}^{i}\right|^{2} \mathrm{~d} r \leq \alpha C_{12}\left(\left\|\hat{W}^{i}\right\|_{K_{M}}^{2}+\varepsilon\right),
\end{array}\right.
$$

where

$$
C_{11}=\frac{C_{10}}{\mu_{1}-\tilde{L}_{\Phi}-8\left(\tilde{L}+\alpha L_{0}+\alpha\right) L_{\Phi}^{2} \mathrm{e}^{\beta T}}, C_{12}=\frac{C_{10}}{\beta_{1}-2\left(\tilde{L}+\alpha L_{0}+\alpha\right)\left(1+2 \mathrm{e}^{\beta T}\right)} .
$$

From the backward SDE of (3.7), we obtain

$$
\begin{aligned}
& E\left[\sup _{t \leq s \leq T}\left|\hat{Y}_{s}^{i}\right|^{2}+\int_{t}^{T}\left|\hat{Z}_{s}^{i}\right|^{2} \mathrm{~d} s\right] \\
\leq & 6 L_{\Phi}^{2} E\left|\hat{X}_{T}^{i}\right|^{2}+\alpha C_{13}\left(\left\|\hat{W}^{i}\right\|_{K_{M}}^{2}+\varepsilon\right)+15 T L^{2} E\left[\int_{t}^{T}\left|\hat{X}_{s}^{i}\right|^{2} \mathrm{~d} s\right]+\left(15 T L^{2}+13\right) E\left[\int_{t}^{T}\left|\hat{Y}_{s}^{i}\right|^{2}+\left|\hat{Z}_{s}^{i}\right|^{2} \mathrm{~d} s\right],
\end{aligned}
$$

where $C_{13}=6 \tilde{L}_{\Phi}^{2} C_{2}+15 T\left(\tilde{L}+\alpha L_{0}\right)^{2} C_{1}+15 T^{2} \alpha$. Finally, combining (A.12), (A.13), (A.17) and (A.18), we conclude that, for $i \geq 0$,

$$
E\left[\sup _{t \leq s \leq T}\left(\left|\hat{X}_{s}^{i}\right|^{2}+\left|\hat{Y}_{s}^{i}\right|^{2}\right)+\int_{t}^{T}\left|\hat{Z}_{s}^{i}\right|^{2} \mathrm{~d} s\right] \leq \alpha C_{14}\left(\left\|\hat{W}^{i}\right\|_{K_{M}}^{2}+\varepsilon\right),
$$

where $C_{14}=\left(15 T L^{2}+13+C_{7}\right)\left(C_{8}+4 L_{\Phi}^{2} \mathrm{e}^{\beta T} C_{11}+2 \mathrm{e}^{\beta T} C_{12}\right)+6 L_{\Phi}^{2} C_{11}+15 T L^{2} C_{12}+C_{13}+C_{6}$.

Acknowledgements. The authors thank the associate editor and the referees for their helpful comments.

\section{REFERENCES}

[1] F. Antonelli, Backward-forward stochastic differential equations. Ann. Appl. Probab. 3 (1993) 777-793.

[2] M. Bossy, Some stochastic particle methods for nonlinear parabolic PDEs. ESAIM: Proc. 15 (2005) $18-57$. 
[3] M. Bossy and D. Talay, A stochastic particle method for the McKean-Vlasov and the Burgers equation. Math. Comput. 66 (1997) 157-192.

[4] R. Buckdahn and J. Li, Stochastic differential games and viscosity solutions of Hamilton-Jacobi-Bellman-Isaacs equations. SIAM J. Control Optim. 47 (2008) 444-475.

[5] R. Buckdahn, J. Li and S.G. Peng, Mean-field backward stochastic differential equations and related partial differential equations. Stoch. Proc. Appl. 119 (2009) 3133-3154.

[6] R. Buckdahn, B. Djehiche, J. Li and S.G. Peng, Mean-field backward stochastic differential equations: A limit approach. Ann. Probab. 37 (2009) 1524-1565.

[7] T. Chan, Dynamics of the McKean-Vlasov equation. Ann. Probab. 22 (1994) 431-441.

[8] M.G. Crandall, H. Ishii and P.L. Lions, User's guide to viscosity solutions of second order partial differential equations. Bull. Amer. Math. Soc. 27 (1992) 1-67.

[9] T. Hao and J. Li, Backward stochastic differential equations coupled with value function and related optimal control problems. Abstr. Appl. Anal. 2014 (2014) 262713.

[10] Y. Hu and S.G. Peng, Solution of forward-backward stochastic differential equations. Probab. Theory Relat. Fields 103 (1995) 273-283.

[11] P. Kotelenez, A class of quasilinear stochastic partial differential equations of McKean-Vlasov type with mass conservation. Probab. Theory Relat. Fields 102 (1995) 159-188.

[12] H. Min, Y. Peng and Y.L. Qin, Fully coupled mean-field forward-backward stochastic differential equations and stochastic maximum principle. Abstr. Appl. Anal. 2014 (2014) 839467.

[13] J. Li and Q.M. Wei, Optimal control problems of fully coupled FBSDEs and viscosity solutions of Hamilton-Jacobi-Bellman equations. SIAM J. Control Optim. 52 (2014) 1622-1662.

[14] J. Li and Q.M. Wei, Stochastic differential games for fully coupled FBSDEs with jumps. Appl Math Optim. 71 (2015) 411-448.

[15] J. Ma, P. Protter and J.M. Yong, Sloving forward-backward stochastic differential equations explicitly-a four step scheme. Probab. Theory Relat. Fields 98 (1994) 339-359.

[16] S.G. Peng, BSDE and Stochastic Optimizations, in Topics on Stochastic Analysis, edited by J.A. Yan, S.G. Peng, S.Z. Fang and L.M. Wu. Science Press, Beijing (1997) 85-138 (in Chinese).

[17] S.G. Peng and Z. Wu, Fully coupled forward-backward stochastic differential equations and applications to optimal control. SIAM J. Control Optim. 37 (1999) 825-843.

[18] P.D. Pra and F.D. Hollander, McKean-Vlasov limit for interacting random processes in random media. J. Stat. Phys. 84 (1996) $735-772$.

[19] D. Talay and O. Vaillant, A stochastic particle method with random weights for the computation of statistical solutions of McKean-Vlasov equations. Ann. Appl. Probab. 13 (2003) 140-180. 